This document is the accepted manuscript version of the following article:

Zhao, Y., Mavrokefalos, C. K., Zhang, P., Erni, R., Li, J., Triana, C. A., \& Patzke, G. R. (2020). self-templating strategies for transition metal sulfide nanoboxes as robust bifunctional

electrocatalysts. Chemistry of Materials. https://doi.org/10.1021/acs.chemmater.9b02933

\title{
Self-Templating Strategies for Transition Metal Sulfide Nanoboxes as Robust Bifunctional Electrocatalysts
}

\author{
Yonggui Zhao ${ }^{1}$, Christos K. Mavrokefalos ${ }^{1}$, Ping Zhang ${ }^{2}$, Rolf Erni ${ }^{3}$, Jingguo Li ${ }^{1}$, C. A. Triana ${ }^{1}$ and \\ Greta R. Patzke ${ }^{1 *}$ \\ ${ }^{1}$ Department of Chemistry, University of Zurich, Winterthurerstrasse 190, CH-8057 Zurich, Switzerland \\ E-mail: greta.patzke@ chem.uzh.ch \\ ${ }^{2}$ School of Electrical and Information Engineering and Key Laboratory of Advanced Ceramics and Machining Technology of \\ Ministry of Education, Tianjin University, Tianjin, 300072, China \\ ${ }^{3}$ Electron Microscopy Center, Empa, Swiss Federal Laboratories for Materials Science and Technology, \\ CH-8600 Dübendorf, Switzerland
}

\begin{abstract}
Hollow nanostructures keep attracting intense interest as multifunctional materials, especially in energy storage and conversion technologies. We introduce a convenient anion exchange method for the synthesis of metal sulfide nanoboxes (NBs) from $\mathrm{Co}, \mathrm{Co}-\mathrm{Fe}$ and Ni-Fe Prussian blue (PB) nanocubes (referred to as Co-PB, Co-Fe-PB and Ni-Fe-PB NCs) as templates. Analytical characterizations show that anionic exchange processes between $\mathrm{S}^{2-}$ and $\mathrm{CN}^{-}$lead to the formation of metal sulfide nanobox heterostructures (referred to as $\mathrm{Co}-\mathrm{S} @ \mathrm{~PB}, \mathrm{Co}-\mathrm{Fe}-\mathrm{S} @ \mathrm{~PB}$, and Ni-Fe-S@PB NBs). The Co-Fe-S $@$ PB NBs were characterized in detail with a wide range of analytical techniques, including X-ray photoelectron spectroscopy (XPS) and X-ray absorption spectroscopy (XAS). Furthermore, post-catalytic XAS and XPS studies indicated that the in situ formation of Co-Fe oxides/hydroxides during the oxygen evolution reaction (OER) provided active species of the Co-Fe-S@PB NBs, while $\mathrm{Fe}(\mathrm{CN})_{6}^{3-}$ did not play a role in the catalytic activity. Together with their advanced morphology, this resulted in a superior OER performance of the as-prepared Co-Fe-S@PB NBs in comparison with $\mathrm{RuO}_{2}$ and other standards. Co-Fe-S@PB NBs excelled through a low overpotential of $286 \mathrm{mV}$ at $10 \mathrm{~mA} / \mathrm{cm}^{2}$, a small Tafel slope value of $37.84 \mathrm{mV} \mathrm{dec} \mathrm{de}^{-1}$ and high durability over the operational period of $33 \mathrm{~h}$ at $10 \mathrm{~mA} / \mathrm{cm}^{2}$. Moreover, we applied our strategy to produce new double-layered $(\mathrm{Co}, \mathrm{Fe})_{9} \mathrm{~S}_{8} @ \mathrm{MoS}_{2}$ nanoboxes (referred to as Co-Fe-Mo-S NBs). The fine-tuned heterostructured nanoboxes are promising for hybrid electrodes due to their high dual OER and hydrogen evolution reaction (HER) activity throughout the $\mathrm{pH}$ range.
\end{abstract}

\section{INTRODUCTION}

Energy consumption of fossil fuels and its inextricable link to climate change has triggered the development of sustainable energy technologies. ${ }^{1-2}$ Electrochemical water splitting is a promising pathway towards clean and storable fuels. Platinumgroup metal (PGM) compounds are regarded as the most active electrocatalysts for overall water splitting, but their high cost and scarcity hinder their practical utilization. ${ }^{3-5}$ Therefore, the development of efficient noble metal-free electrocatalysts is of utmost importance.

Transition metal oxides, ${ }^{6-11}$ (oxy)hydroxides, ${ }^{12-18}$ selenides, ${ }^{19-20}$ sulfides, ${ }^{21-25}$ and phosphides ${ }^{26-30}$ have attracted increasing attention as water splitting catalysts due to their earthabundance and low price. In particular, transition metal sulfides (TMSs) possess significant electrical conductivity and are rich in electroactive redox species so that they are studied as potential electrocatalysts in water splitting. ${ }^{22-24}$ Recent studies confirmed that TMSs contain similar charged states between sulfur and metal sites comparable to hydrogenases. . $224-25$ Sulfur is able to tune the electronic structure of active species and thereby to improve the electrocatalytic activity. ${ }^{22,25}$ Layered molybdenum disulfide $\left(\mathrm{MoS}_{2}\right)$ is one of the most promising TMSs used for the hydrogen evolution reaction (HER). ${ }^{31-32}$ Catalytically active sites of $\mathrm{MoS}_{2}$ were demonstrated to originate from the edges rather than from the basal planes. ${ }^{32-35}$ Therefore, rational design of nanostructured $\mathrm{MoS}_{2}$ with a maximum number of exposed edge sites is an effective strategy to improve its HER activity. While enhanced HER activity of $\mathrm{MoS}_{2}$ could be achieved by morphology control or metal doping to tune its electronic structure, its oxygen evolution reaction (OER) activity is, however, still poor. ${ }^{31-38}$

Rationally-designed hollow nanostructures derived from noble metal-free catalysts are a promising approach to optimize the electrocatalytic activity of TMSs in both HER and OER. ${ }^{20,39-12}$ Hollow nanostructures provide high surface area, expose more edge and active sites and offer a favorable interface between substrate and electrolyte. ${ }^{43-46}$ These synergistic effects of heterogeneous nanostructures often lead to enhanced electrocatalytic activity when compared with their homogeneous counterparts. ${ }^{36-38}$ In most cases, hollow nanostructures are directly prepared via a straightforward hardtemplate method. However, the necessity for pre-designing an appropriate starting template restricts this strategy. This renders self-templated approaches an attractive option, but they remain a challenge for the construction of novel hollow heterogeneous nanostructures of TMS-based materials. On the other hand, Prussian blue analogues (PBAs) are competitive candidates compared to many of the state-of-the-art transition metal oxides when applied in water splitting under neutral conditions. ${ }^{47}$ 
However, their poor conductivity and unstable anionic cyanide groups under alkaline conditions limit their electrocatalytic performance for overall water splitting setups. ${ }^{48-50}$

Herein, we first report on a convenient self-templated method to synthesize new and stable heterogeneous nanobox structures based on $\mathrm{Co}-, \mathrm{Fe}-$ and $\mathrm{Ni}$-sulfides and Prussian blue (CoS@PB, Co-Fe-S@PB and Ni-Fe-S@PB). Our novel synthetic strategy starts from metal-PB nanocubes as a bimetallic selftemplate, followed by its transformation into metal-S@PB NBs utilizing the Kirkendall effect combined with thioacetamide (TAA) as sulfur resource (Figure S2). To the best of our knowledge, our study is the first work which combines the synthesis of nanobox structures with the creation of cyanide vacancies through a PB-based anion exchange process. Moreover, we present new insight into the effect of $\mathrm{Fe}$ leaching on the OER activity. This facile method was furthermore extended to the preparation of double-layered Co-Fe-Mo-S NBs as highly efficient electrocatalysts for both OER and HER. CoFe-S@PB NBs showed superior performance among the series by exhibiting a low overpotential of $286 \mathrm{mV}$ and excellent electrochemical stability for $33 \mathrm{~h}$ without any decline at the constant current density of $10 \mathrm{~mA} / \mathrm{cm}^{2}$ for OER. Concerning IER activity, the elaborate $\mathrm{Co}-\mathrm{Fe}-\mathrm{Mo}-\mathrm{S}$ NBs architectures showed low overpotential of $192 \mathrm{mV}$, which was maintained for over $12 \mathrm{~h}$ at $10 \mathrm{~mA} / \mathrm{cm}^{2}$ under acidic conditions. The nanobox nature of the electrocatalysts was retained before and after electrochemical measurements.

\section{EXPERIMENTAL DETAILS AND METHODS}

Chemicals. Cobalt(II) acetate tetrahydrate ( $\geq 98 \%)$, nickel(II) acetate tetrahydrate ( $\geq 98 \%$ ), potassium hexacyanoferrate(III) ( $\geq 99 \%$ ), potassium hexacyanocobaltate(III) $(\geq 99 \%$ ), trisodium citrate dehydrate $(\geq 99 \%)$, thioacetamide $(\geq 99 \%), \mathrm{NaOH}(\geq 97$ $\%)$, N, N-dimethylformamide ( $\geq 99.98 \%$ ), and KOII ( $\geq 99.97$ $\%)$ were purchased from Sigma- $\Lambda$ ldrich. $\left(\mathrm{NH}_{4}\right)_{2} \mathrm{MoS}_{4}(\geq 99.95$ $\%)$ was purchased from Alfa Aesar. All chemicals used in this study were used as received without any further purification.

Synthesis of Co-PB NCs. Co-PB NCs were prepared as follows: $1.2 \mathrm{mmol}$ of cobalt(II) acetate tetrahydrate and 1.6 mmol of trisodium citrate dihydrate were added into $40 \mathrm{~mL}$ of deionized (DI) water to form a clear transparent pink solution (solution A). $0.8 \mathrm{mmol}$ of potassium hexacyanocobaltate(III) was dissolved in $40 \mathrm{~mL}$ of DI water to form a transparent yellow solution (solution B). Solution B was rapidly added into solution A under magnetic stirring for $3 \mathrm{~min}$. The obtained solution was kept at room temperature for $18 \mathrm{~h}$. The precipitate was collected by centrifugation, washed 3 times with DI water and dried at $70^{\circ} \mathrm{C}$ overnight.

Synthesis of Co-Fe-PB NCs. Co-Fe-PB NCs were prepared as follows: $1.2 \mathrm{mmol}$ of cobalt(II) acetate tetrahydrate and 1.5 mmol of trisodium citrate dihydrate were added into $40 \mathrm{~mL}$ of deionized (DI) water to form a clear transparent pink solution (solution A). $0.8 \mathrm{mmol}$ of potassium hexacyanoferrate(III) was dissolved in $60 \mathrm{~mL}$ of DI water to form a transparent yellow solution (solution B). Solution B was rapidly added into solution A under magnetic stirring for $2 \mathrm{~min}$. The obtained solution was aged at room temperature for $24 \mathrm{~h}$. The precipitate was collected by centrifugation, washed 3 times with DI water and dried at $70{ }^{\circ} \mathrm{C}$ overnight.
Synthesis of Ni-Fe-PB NCs. Ni-Fe-PB NCs were prepared as follows: $1.2 \mathrm{mmol}$ of nickel(II) acetate tetrahydrate and 1.5 mmol of trisodium citrate dihydrate were added into $40 \mathrm{~mL}$ of deionized (DI) water to form a clear transparent green solution (solution A). $0.8 \mathrm{mmol}$ of potassium hexacyanoferrate(III) was dissolved in $60 \mathrm{~mL}$ of DI water to form a transparent yellow solution (solution B). Solution B was rapidly added into solution $\Lambda$ under magnetic stirring for $3 \mathrm{~min}$. The obtained solution was aged at room temperature for $48 \mathrm{~h}$. The precipitate was collected by centrifugation, washed 3 times with DI water and dried at $70{ }^{\circ} \mathrm{C}$ overnight.

Synthesis of Co-S@PB, Co-Fe-S@PB, Ni-Fe-S@PB NBs. In a typical synthetic procedure, $20 \mathrm{mg}$ of $\mathrm{Co} / \mathrm{Co}-\mathrm{Fe} / \mathrm{Ni}-\mathrm{Fe}-\mathrm{PB} \mathrm{NC}$ precursors, respectively, were dispersed in $6 \mathrm{~mL}$ of ethanol by sonication for $30 \mathrm{~min}$ to obtain a very homogeneous dispersion. $50 \mathrm{mg}$ of thioacetamide (TAA) were dissolved into $6 \mathrm{~mL}$ of ethanol to obtain a transparent solution. The TAA solution was then injected into the above dispersion rapidly. After continuous stirring for $10 \mathrm{~min}$, the mixture was transferred into a $20 \mathrm{~mL}$ Teflon-lined stainless steel autoclave and kept at $160{ }^{\circ} \mathrm{C}$ for different reaction times. The resultants were collected by centrifugation at $5000 \mathrm{rpm} / \mathrm{min}$ and washed with ethanol several times. The obtained nanoboxes were dried at room temperature. The synthesis of $\mathrm{Co}-\mathrm{Fe}-\mathrm{S} @ \mathrm{~PB}$ nanoparticles was performed as in the aforementioned procedure. However, the reaction was conducted at different temperatures and reaction times.

Synthesis of Co-Fe oxides. To obtain the reference Co-Fe oxides, the as-prepared $\mathrm{Co}-\mathrm{Fe} \mathrm{NBs}$ precursor was calcined at $350{ }^{\circ} \mathrm{C}$ in the air for $2 \mathrm{~h}$ with a ramping rate of $2{ }^{\circ} \mathrm{C} / \mathrm{min}$.

Synthesis of $(\mathrm{Co} / \mathrm{Co}-\mathrm{Fe})(\mathrm{OH})_{x}$ NBs. $(\mathrm{Co} / \mathrm{Co}-\mathrm{Fe})(\mathrm{OH})_{x} \mathrm{NBs}$ were prepared as follows: $45 \mathrm{mg} \mathrm{Co} / \mathrm{Co}-\mathrm{Fe} \mathrm{NC}$ precursor was dispersed in $30 \mathrm{~mL}$ of ethanol and sonicated for $15 \mathrm{~min}$ to form a homogeneous suspension. $15 \mathrm{~mL}$ of $2 \mathrm{M} \mathrm{NaOH}$ solution was then added to the above suspension and stirred for $20 \mathrm{~min}$. The obtained precipitation was collected by centrifugation, washed 3 times with DI water and ethanol and dried at $70^{\circ} \mathrm{C}$ overnight.

Synthesis of double-shell Co-Fe-Mo-S NBs. In a typical synthetic procedure, $60 \mathrm{mg}$ of $\mathrm{Co}-\mathrm{Fe}-\mathrm{PB} \mathrm{NCs}$ and $20 \mathrm{mg}$ of $\left(\mathrm{NH}_{4}\right)_{2} \mathrm{MoS}_{4}$ were dispersed in $60 \mathrm{~mL}$ of $\mathrm{N}, \mathrm{N}-$ dimethylformamide (DMF) via sonication for $20 \mathrm{~min}$ to obtain a very homogeneous dispersion. The mixture was then transferred into $90 \mathrm{~mL}$ Teflon-lined stainless steel autoclave and kept at $210{ }^{\circ} \mathrm{C}$ for different reaction times $(2 \mathrm{~h}, 12 \mathrm{~h}, 20 \mathrm{~h}$, and $24 \mathrm{~h}$ ). The resulting products were collected by centrifugation at $5000 \mathrm{rpm} / \mathrm{min}$ for $10 \mathrm{~min}$ and washed several times with ethanol and DI water. The obtained Co-Fe-Mo-S NBs were dried at $70^{\circ} \mathrm{C}$ overnight.

Furthermore, pure $\mathrm{MoS}_{2}$ nanosheets were prepared as a reference. The synthesis of $\mathrm{MoS}_{2}$ nanosheets was similar to the synthesis of Co-Fe-Mo-S NBs, apart from the absence of Co$\mathrm{Fe}-\mathrm{PB} \mathrm{NC}$ precursor during the synthesis.

Analytical characterization. X-ray diffraction (XRD) patterns were collected on a STOE STADI P diffractometer (transmission mode, Ge monochromator) with $\mathrm{Mo} \mathrm{K}_{\alpha}$ radiation $(\lambda=0.7903 \AA)$ at a voltage of $50 \mathrm{kV}$ and current of $40 \mathrm{~mA}$. The microstructures were characterized with transmission electron 
microscopy (TEM; Hitachi HT7700 EXALENS), and fieldemission scanning electron microscopy (FESEM; Zeiss Supra $50 \mathrm{VP}$ ). Energy-dispersive X-ray spectroscopy (EDX) attached to the FESEM instrument was employed for the compositional analysis of the samples. High-resolution scanning transmission electron microscopy (HR-STEM), high angle annular dark-field scanning transmission electron microscopy (HAADF-STEM) images, and STEM-EDX elemental maps were obtained with an FEI Titan Themis equipped with a hexapole-type aberration corrector for scanning transmission electron microscope (CEOS DCOR) and a super EDX system. Attenuated total reflectance Fourier-transform infrared (ATR-FT-IR) spectra were recorded on a Bruker Vertex 70 spectrometer equipped with a Platinum ATR accessory containing a diamond crystal. Raman spectra were measured using a Renishaw Raman scope or InVia Qontor (Ar+ laser, $514 \mathrm{~nm}$ ) for pristine powder samples on quartz glass slides. Brunauer-Emmett-Teller surface area measurements were carried out after degassing the samples at $150{ }^{\circ} \mathrm{C}$ for $20 \mathrm{~h}$ under vacuum using a Quantachrome Quadrasorb SI porosimeter at $77 \mathrm{~K}$. Adsorption branch points $(0.05<\mathrm{p} / \mathrm{p} 0<$ 0.3) were applied in the BET model to calculate the apparent surface area. An ESCALAB 250XI instrument with Al $\mathrm{K}_{\alpha}$ $(1486.6 \mathrm{eV})$ radiation operated at $250 \mathrm{~W}$ was employed for XPS studies. X-ray-absorption near-edge structure (XANES) and extended X-ray-absorption fine structure (EXAFS) experiments at the $\mathrm{Co}$ and $\mathrm{Fe} \mathrm{K}$-edges on solid samples dispersed in cellulose of pristine and post-catalytic of samples were carried out at the European Synchrotron Radiation Facility (ESRF), SwissNorwegian Beamline BM31, Grenoble, France.

\section{RESULTS AND DISCUSSION}

\section{Synthesis of Co-Fe-PB NC templates}

The binary metal template Co-Fe-PB NCs was prepared through a co-precipitation method (see experimental details). PXRD patterns (Figure S3a) of the obtained Co-Fe-PB NCs showed that all the diffraction peaks match with the standard cubic structure of $\mathrm{Co}_{3}\left[\mathrm{Fe}(\mathrm{CN})_{6}\right]_{2} \cdot 10 \mathrm{H}_{2} \mathrm{O}$ (PDF\#86-0502). Furthermore, EDX results (Figure $\mathrm{S} 3 \mathrm{~b}$ ) indicated a $\mathrm{Co} / \mathrm{Fe}$ atomic ratio of $1.45: 1$, which is comparable to the desired ratio of 1.5:1. Field-emission scanning electron microscopy (FESEM) and transmission electron microscopy (TEM, Figure S4) showed the cubic nature of the as-prepared Co-Fe-PB with an average size between 200 and $300 \mathrm{~nm}$. The structural features of Co-Fe-PB NCs of our study are in agreement with previous literature reports. ${ }^{43}$

\section{Control of the morphological evolution}

In order to obtain stable Co-Fe-S@PB hollow structures for optimal electrocatalytic properties, time-dependent experiments (Figure 1 and Figure S5) were conducted to monitor their morphological evolution. Figures 1a, e and i illustrate the uniform morphological distribution of the Co-FePB template cubes. After sulfidation at $160{ }^{\circ} \mathrm{C}$ between 2 and $10 \mathrm{~h}$ (Figures 1b-c, f-g, j-k, and Figures S5b-e), the obtained $\mathrm{Co}-\mathrm{Fe}-\mathrm{S}(a) \mathrm{PB}$ products retained their cubic shapes along with their rough surface and empty cavities, suggesting the successful formation of hollow structures. When the reaction time was prolonged to $15 \mathrm{~h}$, the nanobox structures collapsed (Figures 1d, h, 1 and Figure S5f). First, the long-lasting supply of $\mathrm{S}^{2-}$ is a crucial parameter for the morphological evolution of the Co-Fe-S@PB NBs. Controlling the amount of TAA is another important parameter for the formation of hollow structures. In particular, nanobox morphologies with a thick shell were obtained with a low amount of TAA, namely $20 \mathrm{mg}$ (Figures S6a, c, e). After the amount of TAA was increased to $100 \mathrm{mg}$, similar nanobox morphologies with thin layers were prepared, however, part of these structures was disintegrating (Figures S6b, d, f). The low amount of TAA slowed down the diffusion rate of $\mathrm{S}^{2-}$ ions and resulted in a thick shell. In contrast, increase of the TAA amount most likely accelerated the anionic exchange process and resulted in hollow structures at an early stage, followed by their collapse due to the continuing diffusion effects. Analogous results were obtained when the reaction temperature was increased from $120^{\circ} \mathrm{C}$ to $180^{\circ} \mathrm{C}$ (Figure S7). The obtained products exhibited a nanocubic structure at lower temperature (Figures S7a-b). The nanocubic structure was preserved but covered by smaller particles when sulfidation was operated at $180^{\circ} \mathrm{C}$ (Figure S7c). In addition to TAA amount and reaction temperature, other parameters also played an important role. When replacing ethanol by isopropanol, a uniform nanobox morphology with thicker shell was obtained (Figures S8a, c, e). However, synthesis in deionized water (Figures $\mathrm{S} 8 \mathrm{~b}, \mathrm{~d}, \mathrm{f}$ ) did not preserve the desired nanobox morphology of the final products. This is due to $\mathrm{OH}^{-}$diffusion processes, which compete with the anion exchange reaction of $\mathrm{OH}^{-}$and those of $\mathrm{S}^{2-}$ and $\mathrm{CN}^{-}$, respectively. These observations are in agreement with the schematic formation mechanism proposed in Figure S2.

\section{Characterization of Co-Fe-S $a$ PB NBs}

The PXRD patterns (Figure 2a) of the Co-Fe-PB NCs obtained after various reaction times showed that the crystal structures of the as-prepared samples match perfectly with the pristine template of Co-Fe-PB NCs (reaction time $\leq 10 \mathrm{~h}$ ). HR-STEM images and S@PB NBs (10 h). The HR-STEM of the outer layer showed a lattice distance of $5.2 \AA$, which is assigned to $\{200\}$ planes of Co-Fe- PB NCs. No such information was obtained for the $(\mathrm{Co}, \mathrm{Fe}) \mathrm{S}_{\mathrm{x}}$ surface layer, thus indicating its amorphous features. Acquisition of HR-STEM images for Co-

Figure 1. FESEM and TEM images of as-prepared samples. (a, e, i) Co-Fe-PB NCs; Co-Fe-S@PB NBs obtained at $160{ }^{\circ} \mathrm{C}$ at different sulfidation durations: (b, f, j) $2 \mathrm{~h},(\mathrm{c}, \mathrm{g}, \mathrm{k}) 10 \mathrm{~h},(\mathrm{~d}, \mathrm{~h}, \mathrm{l}) 15 \mathrm{~h}$. (m) High-resolution scanning transmission electron microscopy (HR-STEM) and selected area electron diffraction (SAED) images of Co-Fe-S@PB NBs at $160^{\circ} \mathrm{C}$ after 10 h. (n) High-angle annular dark-field scanning transmission electron microscopy (HAADF-STEM) images and STEM-EDX elemental maps of Co-Fe-S $@$ ) PB NBs at $160{ }^{\circ} \mathrm{C}$ after $10 \mathrm{~h}$ (cyan: C, yellow: $\mathrm{N}$, red: Co, green: Fe, blue: $\mathrm{S}$ ).

Figure 2. Structural characterizations of Co-Fe-S $($,PB catalysts obtained after various sulfidation times. (a, b) XRD patterns and FT-IR spectra of Co-Fe-S@PB NBs at various sulfidation times. (c) Co K-edge XANES of Co-Fe-S@PB at various sulfidation times vs. references. (d) FT-EXAFS of $\mathrm{Co}$ in $\mathrm{Co}-\mathrm{Fe}-\mathrm{S} @ \mathrm{~PB}$ at various sulfidation times. (e) High resolution XPS spectra of Co, Fe, and $\mathrm{S}$ for $\mathrm{Co}-\mathrm{Fe}-\mathrm{PB} \mathrm{NCs}$, CoFe-S@PB NBs, and Co-Fe-S@PB particles. 
Fe-S@PB NBs is challenging because the boxes are large, and the projection along the wall direction is thus quite thick. Nevertheless, HR-TEM diffraction images (Figures S9-10) were recorded and they revealed that introduction of $\mathrm{S}$ does not change the crystallinity of pristine $\mathrm{Co}-\mathrm{Fe}-\mathrm{PB}$, while the amorphous structure of the outer layer arises from the Co-Fe-S phase. Elemental mapping revealed the distribution of $\mathrm{Co}, \mathrm{Fe}$, $\mathrm{C}$, and $\mathrm{N}$ over both the inner and outer layers of the NBs, while $\mathrm{S}$ was uniformly dispersed around the outer layer (Figure 1), indicating that the diffusion process indeed proceeded from outside to inside (Figure S2). When the reaction time was extended to $15 \mathrm{~h}$, impurities were detected in the PXRD pattern, which are attributed to $(\mathrm{Co}, \mathrm{Fe}) \mathrm{S}_{\mathrm{x}}$. EDX results (Figures S1112) showed that the atomic ratio of $\mathrm{Co} / \mathrm{Fe}$ did not significantly change as a function of reaction time, while an increasing trend was found for the atomic ratio of $\mathrm{S} /(\mathrm{Co}, \mathrm{Fe})$. The $\mathrm{S}^{2-} / \mathrm{CN}^{-}$ exchange was furthermore investigated with elemental analysis (Table S1) of Co-Fe-PB NCs obtained after reaction times of 0 , 10 and $15 \mathrm{~h}$. While the $\mathrm{C}$ and $\mathrm{N}$ contents decreased, the amount of $\mathrm{S}$ increased from 0 to $29 \%$ for the Co-Fe-PB NCs from 0 to $15 \mathrm{~h}$, respectively.

The synthesis of porous cobalt sulfide NCs under aqueous conditions was reported in previous works, where the anion exchange reaction was found to occur between $\mathrm{Fe}(\mathrm{CN})_{6}{ }^{3-}$ in $\mathrm{Co}-$ Fe-PB and $\mathrm{S}^{2-}$ from $\mathrm{Na}_{2} \mathrm{~S}$, respectively. ${ }^{51-52}$ At the end of the reaction, no $\mathrm{Fe}$ species remained in the final samples. In contrast, we found here that the ratio of $\mathrm{Co}$ and Fe remained proportional before and after the reaction. Hence, the major anion exchange reaction during the sulfidation process is based on chemical diffusion of $\mathrm{S}^{2-}$ and $\mathrm{CN}^{-}$. The IR spectra of the products after different reaction times display two peaks in the range of 2000 and $2200 \mathrm{~cm}^{-1}$, a weak peak at $2117 \mathrm{~cm}^{-1}$ and a stronger peak at $2087 \mathrm{~cm}^{-1}$ (Figure 2b). The former peaks at $2117 \mathrm{~cm}^{-1}$ appeared when $\mathrm{K}^{+}$ions were included in the compounds, and they are assigned to the stretching mode of $\mathrm{CN}$ groups in $\mathrm{Co}^{\mathrm{III}}-\mathrm{NC}-\mathrm{Fe}^{\mathrm{II}}$, adjacent to the potassium ions. ${ }^{53}$ The second peak at $2087 \mathrm{~cm}^{-1}$ is ascribed to the $\mathrm{CN}$ stretching mode of the $\mathrm{Fe}^{\mathrm{II}}-\mathrm{CN}-\mathrm{Co}^{\mathrm{II}}$ moieties, which are more remote from the $\mathrm{K}^{+}$ions. As the reaction time increased from 0 to $15 \mathrm{~h}$, the $v(\mathrm{CN})$ peak at $2117 \mathrm{~cm}^{-1}$ disappeared, whereas the peak at 2087 $\mathrm{cm}^{-1}$ remained. This indicates that the sulfide ions mainly replaced the cyanide groups of the $\mathrm{Co}{ }^{\mathrm{III}}-\mathrm{NC}-\mathrm{Fe}^{\mathrm{II}}$ chains around the $\mathrm{K}^{+}$ions. ${ }^{53}$ To further study the sulfide exchange reaction, XAS measurements were employed to provide insight into the individual elemental coordination environments. ${ }^{54-56}$ Figure $2 \mathrm{c}$ depicts the Co K-edge X-ray absorption near-edge structure (XANES) spectra of the template samples (Co-Fe-PB NCs), of samples after $10 \mathrm{~h}(\mathrm{Co}-\mathrm{Fe}-\mathrm{S} @ \mathrm{~PB} \mathrm{NBs})$ and $15 \mathrm{~h}$ of reaction time (Co-Fe-S@PB particles), respectively, as well as of reference samples. Clearly, the Co-Fe-S@PB NBs and the CoFe-S@PB particles showed similar line shapes in their Co Kedge XANES spectra, indicating that the local electronic structure of the cobalt ions remained more or less unchanged after sulfidation. Compared to Co-Fe-PB NCs, a negative shift of the Co K-edge position was observed for the Co-Fe-S@PB NBs and Co-Fe-S@PB particles, suggesting that the oxidation state of cobalt ions was mainly changed to $\mathrm{Co}^{2+}$ after sulfur was introduced. This observation was also consistent with the IR spectra, where the $v(\mathrm{CN})$ peak of $\mathrm{Co}^{\mathrm{III}}-\mathrm{NC}-\mathrm{Fe}^{\mathrm{II}}$ at $2117 \mathrm{~cm}^{-1}$ disappeared after sulfur addition. The Fourier-transform (FT) $\mathrm{k}^{3}$-weighted extended X-ray absorption fine structure (FTEXAFS) spectra also showed two prominent peaks at $1.90 \AA$ for the $\mathrm{Co}-\mathrm{N} / \mathrm{O}$ bond and another peak at $2.45 \AA$ for the Co-C bond ${ }^{54}$ However, after treatment with sulfur, a new peak at 1.69 $\AA$ was observed and attributed to $\mathrm{Co}-\mathrm{S}$ bond formation. ${ }^{55-56}$ Furthermore, XANES and FT-EXAFS spectra at the Fe K-edge (Figures S13a-b) showed analogous effects to those of the Co $\mathrm{K}$-edge. More precisely, a significant scattering peak of both Co-Fe-S@PB NBs and Co-Fe-S@PB particles at $1.81 \AA$ was observed and assigned to the Fe-S bond. ${ }^{54}$ The above XAS results provide further strong evidence for the surface exchange reaction between $\mathrm{S}^{2-}$ and $\mathrm{CN}^{-}$.

Surface oxidation state and chemical composition analyses of the as-prepared $\mathrm{Co}-\mathrm{Fe}-\mathrm{S} @ \mathrm{~PB}$ products were further conducted with XPS (Figure 2e and Figures S14-15). As shown in Figure $2 \mathrm{e}$, the Co $2 \mathrm{p}$ signals of the Co-Fe-PB NCs with Co $2 \mathrm{p}_{3 / 2}$ and Co $2 \mathrm{p}_{1 / 2}$ peaks at $782.7 \mathrm{eV}$ and $797.7 \mathrm{eV}$, respectively, are attributed to low-spin $\mathrm{Co}^{3+}$ with $15 \mathrm{eV}$ difference in binding energy. ${ }^{57-58}$ Obviously, after sulfidation occurred, some new peaks appeared at around $779.6 \mathrm{eV}$ near the $\mathrm{Co} 2 \mathrm{p}_{3 / 2}$ peak in the spectra of the Co-Fe-S@PB NBs and Co-Fe-S@PB particles, respectively, which both are assigned to metal sulfide bonds. ${ }^{54}$ ${ }^{55}$ Furthermore, two sharp peaks located around $708.8 \mathrm{eV}(\mathrm{Fe}$ $\left.2 p_{3 / 2}\right)$ and $721.7 \mathrm{eV}\left(\mathrm{Fe} 2 \mathrm{p}_{1 / 2}\right.$, Figure S15) are attributed to the $\mathrm{Fe}(\mathrm{CN})_{6}{ }^{3-}$ moieties ${ }^{54}$ The latter peaks in the as-prepared Co-Fe$\mathrm{S}(\mathrm{PB}$ samples indicate similar coordination environments around Fe. Moreover, XPS results for the Co $2 p$ and Fe $2 p$ peak regions are in agreement with the XAS spectra (Figure $2 \mathrm{c}$ and Figure S13a). In the high-resolution XPS spectrum of S $2 p$, the binding energies at $162.9 \mathrm{eV}$ and $164.2 \mathrm{eV}$ are ascribed to $\mathrm{S}^{2-}$ $2 p_{3 / 2}$ and $S^{2-} 2 p_{1 / 2}$, respectively. Of note is that partial surface oxidation by air resulted in the formation of higher oxidized $\mathrm{SO}_{4}{ }^{2-}$ species with a peak around $169 \mathrm{eV}$, as reported in previous studies. ${ }^{22-25}$

\section{Electrochemical water splitting activity}

To evaluate their application potential, cyclic voltammetry (CV) measurements of the as-prepared Co-Fe-S@PB products as anodic materials were performed in $1 \mathrm{M} \mathrm{KOH}$ solution at $\mathrm{pH}$ 13.6 (Figure 3 and Figure S16) for samples with different morphologies (Figure 1, cf. Co-Fe-S@PB NBs at 10 h, Co-Fe$\mathrm{S}(\mathrm{PB}$ particles at $15 \mathrm{~h}$ ). The advantages of the as-prepared CoFe-S@PB NBs were compared to various references, namely Co-Fe-PB NCs, Co-Fe oxides (Figure S17), Co-Fe-S@PB particles, commercial $\mathrm{Co}(\mathrm{OHI})_{2}, \mathrm{RuO}_{2}$, and $\mathrm{IrO}_{2}$. iR-ohmic drop (75\%) corrections were applied to all LSV measurements in order to reflect the intrinsic behavior of anodic materials (Figure 3a and Figure S18). As shown in Figures 3a-c, Co-Fe-S-@PB NBs display a quite low overpotential value of $286 \mathrm{mV}$ at the current density of $10 \mathrm{~mA} / \mathrm{cm}^{2}$ with a high mass activity of $112.45 \mathrm{~N} / \mathrm{g}$ at the overpotential of $300 \mathrm{mV}$. They outperformed reference $\mathrm{Co}-\mathrm{Fe}$ oxides $(443 \mathrm{mV}, 1.85 \mathrm{~N} / \mathrm{g})$ as well as commercial $\mathrm{Co}(\mathrm{OH})_{2}(403 \mathrm{mV}, 1.55 \mathrm{~N} / \mathrm{g}), \mathrm{RuO}_{2}(344 \mathrm{mV}$, $17.61 \mathrm{~A} / \mathrm{g})$, and $\mathrm{IrO}_{2}(442 \mathrm{mV}, 2.62 \mathrm{~A} / \mathrm{g})$. An increase in OER activity from Co-Fe-PB NCs to Co-Fe-S@PB particles and

Figure 3. (a) LSV curves with scan rate of $5 \mathrm{mV} / \mathrm{s}$ for Co-Fe-PB NCs, Co-Fe-S $@$ PB NBs $(10 \mathrm{~h}), \mathrm{Co}-\mathrm{Fe}-\mathrm{S} @ \mathrm{~PB}$ particles $(15 \mathrm{~h})$, and Co-Fe oxides compared with commercial $\mathrm{Co}(\mathrm{OH})_{2}, \mathrm{RuO}_{2}$, and $\mathrm{IrO}_{2}$ (Sigma) in $1 \mathrm{M} \mathrm{KOH}$. (b) Overpotential required for $\mathrm{j}=1$ and $10 \mathrm{~mA} \mathrm{~cm}{ }^{-2}$. (c) Mass activities at an overpotential of $300 \mathrm{mV}$. (d, e) Tafel plots and Nyquist plots (at an overpotential of $300 \mathrm{mV}$ ) for the six catalysts. (f) Current density vs. scan rate. 
subsequently to Co-Fe-S@PB NBs demonstrated the superior electrocatalytic properties of the latter.

Importantly, the OER performance of Co-Fe-S $a$, PB NBs is competitive with the reported OER of various key materials (Table S2). The turnover frequencies (TOF) were calculated under the assumption that all metal sites are active during the OER process (see Supporting Information for TOF calculations). In particular, the Co-Fe-S@PB NBs showed the best TOF value of $1.03 \mathrm{~s}^{-1}$, higher than the Co-Fe-PB NCs $(0.02$ $\left.\mathrm{s}^{-1}\right)$ and Co-Fe-S $@$ PB particles $\left(0.44 \mathrm{~s}^{-1}\right)$ at an overpotential of $300 \mathrm{mV}$. Tafel plots were further investigated to obtain an insight into the kinetic mechanism. As shown in Figure $3 \mathrm{~d}$, the Co-Fe-S $($ PB NBs exhibited the lowest Tafel slope value of $37.84 \mathrm{mV} \mathrm{dec}^{-1}$ compared to Co-Fe-S $@$ PB particles $(42.08 \mathrm{mV}$ $\left.\mathrm{dec}^{-1}\right), \mathrm{Co}(\mathrm{OH})_{2}\left(63.08 \mathrm{mV} \mathrm{dec}{ }^{-1}\right), \mathrm{RuO}_{2}\left(72.64 \mathrm{mV} \mathrm{dec}{ }^{-1}\right), \mathrm{Co}-$ Fe-PB NCs $\left(78.25 \mathrm{mV} \mathrm{dec}{ }^{1}\right), \mathrm{Co}-\mathrm{Fe}$ oxides $\left(86.88 \mathrm{mV} \mathrm{dec}^{-1}\right)$, and $\mathrm{IrO}_{2}\left(88.16 \mathrm{mV} \mathrm{dec}^{-1}\right)$. In addition, electrochemical impedance spectroscopy (EIS) measurements at the overpotential of $300 \mathrm{mV}$ clearly showed that the Co-Fe-S@PB NBs had a smaller charge transfer resistance $R_{c t}$ (Figure $3 e$ ). The lower $R_{c t}$ value of $23.6 \Omega$ of the Co-Fe-S $@$ PB NBs is attributed to a faster built-in electron conductive process during OER. ${ }^{40}$ The activity measurements in their entirety suggest that the CoFe-S@PB NBs are more efficient compared to Co-Fe-PB NCs due to the higher electronic conductivity of metal sulfides. ${ }^{36-38}$ Moreover, the benefits of the nanobox structures are shortened diffusion lengths of electrons, facilitated penetration of electrolyte and easier release of produced oxygen gas, ${ }^{43-45}$ which all result in a remarkably low overpotential for the OER.

The electrochemical surface area (ECSA) was measured by calculating first the electrochemical double-layer capacitance $\left(\mathrm{C}_{\mathrm{dl}}\right)$. Several CVs were performed in a small potential window between $1.261 \mathrm{~V}$ and 1.361 V (vs. RHE) without any Faradaic process involved (Figure S19). The following sequence of the $\mathrm{C}_{\mathrm{dl}}$ values was observed (Figure 3f): Co-Fe-S@PB NBs (26.51

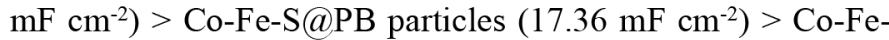
PB NCs $\left(3.21 \mathrm{mF} \mathrm{cm}^{-2}\right)$, indicating that the Co-Fe-S@PB NBs possess higher surface area due to their hollow features. Furthermore, BET-surface areas of the Co-Fe-PB NCs, Co-Fe$\mathrm{S}(a \mathrm{~PB}$ NBs and Co-Fe-S $(\mathrm{PB}$ particles were determined as $33.3 \mathrm{~m}^{2} / \mathrm{g}, 45.0 \mathrm{~m}^{2} / \mathrm{g}$ and $38.5 \mathrm{~m}^{2} / \mathrm{g}$, respectively (Table S3). Obviously, the Co-Fe-S $($ PB NBs also showed the highest BET surface area, consistent with the ECSA results (Figure 3f). The open and hollow box-like structures improved the contact at the interface between the catalyst and electrolyte, allowing for better gas production at the electrode. Therefore, more exposed active sites were obtained in Co-Fe-S@PB NBs and further improved the OER activity. The geometric effects of the electrode were excluded as discussed in literature. ${ }^{45}$ The compared normalized current densities (Figure S20) further demonstrated the intrinsic enhancement of the catalytic activity in Co-Fe-S@PB NBs. ${ }^{25}$ A schematic representation of the RRDE technique is given as inset in Figure 4a. A potential of $0.4 \mathrm{~V}$ (vs. RHE) was applied in the ring electrode to reduce the generated $\mathrm{O}_{2}$ from the disk electrode. A constant current of 250 $\mu \mathrm{A}$ was applied to the disk electrode to generate $\mathrm{O}_{2}$ and maintained for $300 \mathrm{~s}$. A relatively stable current response of 48 $\mu \mathrm{A}$ was detected from the ring electrode. The calculated Faradaic efficiency of $98 \%$ (see the Supporting Information for Faradaic efficiency calculation) verified that the observed current is indeed related to the OER.

The electrochemical stability of the as-prepared catalysts was investigated with a chronopotentiometric method. As shown in Figure $4 \mathrm{~b}$ and Figure S21, the Co-Fe-S@PB NBs exhibited excellent stability under the constant current densities of 5 and $10 \mathrm{~mA} / \mathrm{cm}^{2}$ and maintained their initial potential with only 3 $\mathrm{mV}$ increase after $6 \mathrm{~h}$. The Co-Fe-S@PB NBs outperformed the Co-Fe-S@PB particles $(23 \mathrm{mV})$ as well as commercial $\mathrm{Co}(\mathrm{OH})_{2}(10 \mathrm{mV}), \mathrm{RuO}_{2}(66 \mathrm{mV})$, and $\mathrm{IrO}_{2}$ (over $90 \mathrm{mV}$ ). Of note is that all the measurements indicated that the catalysts with nanobox features maintained high activity and good stability. In general, the prepared layer on the GC electrode was not stable when operated under very long-time measurements and high rotation speed. Herein, the Co-Fe-S $a$, PB NBs were coated with the same mass loading on $\mathrm{Ni}$ foam. The initial current density (Figure 4c) was retained without any current drop after $33 \mathrm{~h}$. Moreover, LSV curves obtained before and after longtime measurements further revealed the excellent operational stability of Co-Fe-S@PB NBs (Figure 4c). Poststability morphological characterizations of the Co-Fe-S@PB NBs confirmed that the nanobox structures retained their initial morphology as illustrated by FESEM and TEM (Figure S22). These results demonstrated the superior activity and stability of Co-Fe-S $\propto$ PB NBs compared to commercial $\mathrm{RuO}_{2}$, which renders them very promising noble metal-free candidates for future electrocatalytic oxygen evolution applications.

\section{Active species and reaction pathways of Co-Fe-S $($ PB NBs}

The active species behind the high activity and excellent OER stability of the newly synthesized Co-Fe-S $(a)$ PB NBs were further investigated. Prior to the electrochemical performance studies, multiple CVs were tested in different conditions (KOH (1) containing the leached Fe species and fresh $\mathrm{KOH}(2), \mathrm{cf}$. Figure S27 and Supplementary Note on CV tests). During the electrochemical measurements, leaching exchange between $\mathrm{Fe}(\mathrm{CN})_{6}{ }^{3-}$ and $\mathrm{OH}^{-}$was observed for the Co-Fe-S@PB NBs (Figures S27-29). To verify the leaching effects of $\mathrm{Fe}(\mathrm{CN})_{6}^{3-}$ into the $\mathrm{KOH}$ solution, LSV curves of $\mathrm{Ni}$ foam combined with addition of different amounts of $\mathrm{K}_{3} \mathrm{Fe}(\mathrm{CN})_{6}$ were recorded as shown in Figure 5a. For the as-synthesized nanoboxes, the leached amounts of $\mathrm{Fe}(\mathrm{CN})_{6}{ }^{3-}$ were found in the region of the red line related to the mass loading of Co-Fe-S@PB NBs on Ni foam (Figure S30 and ICP results in Table S4). In comparison with $\mathrm{Ni}$ foams combined with different amounts of $\mathrm{K}_{3} \mathrm{Fe}(\mathrm{CN})_{6}$, the hybrid system of Co-Fe-S@PB NBs with Ni foam showed the highest current density and best activity at a potential of 1.6 $\mathrm{V}$ vs. RHE. Therefore, $\mathrm{Fe}(\mathrm{CN})_{6}{ }^{3-}$ did not contribute to the OER activity under alkaline conditions. Further control experiments without $\mathrm{Fe}$ (see $\mathrm{Co}-\mathrm{S}(\mathrm{a}) \mathrm{PB} \mathrm{NBs}$ ) were carried out to investigate the role of $\mathrm{Fe}$ in the OER activity (Figure 5b). PXRD patterns,

Figure 4. (a) Faradaic efficiency measurements based on the RRDE technique. (b) Chronopotentiometric measurements of Co-Fe-S@PB catalyst at current densities of 5 and $10 \mathrm{~mA} \mathrm{~cm}{ }^{-2}$. (c) Stability measurements of the Co-Fe-S(a)PB NBs loaded on Ni foam.

Figure 5. (a) LSV curves of blank Ni foam with different amounts of $\mathrm{K}_{3}\left[\mathrm{Fe}(\mathrm{CN})_{6}\right]$ during OER. (b) Electrochemical performance (LSV curves) for catalysts before and after rinsing with $2 \mathrm{M} \mathrm{NaOH}$. (c) Overpotential required for $10 \mathrm{~mA} \mathrm{~cm}$. 
FT-IR, and EDX spectra (Figures S31-32) confirmed that similar anion exchange processes between $\mathrm{CN}^{-}$and $\mathrm{S}^{2-}$ took place in reference Co-PB templates. EDX data showed that the $\mathrm{S} / \mathrm{Co}$ atomic ratio of the $\mathrm{Co}-\mathrm{S} @ \mathrm{~PB} \mathrm{NBs}$ was approximately 0.8 , similar to the $\mathrm{S} /(\mathrm{Co}, \mathrm{Fe})$ atomic ratio of the Co-Fe-S@PB NBs. Furthermore, TEM images of Figure S33 depicted that Co$\mathrm{S} @$ PB NBs were obtained when the Co-PB NCs template was sulfidized for $24 \mathrm{~h}$. $\Lambda$ s shown in Figures $5 \mathrm{~b}-\mathrm{c}$, a lower overpotential of the Co-Fe-S@PB NBs at $10 \mathrm{~mA} / \mathrm{cm}^{2}$ was obtained compared to iron-free $\mathrm{Co}-\mathrm{S} @ \mathrm{~PB}$ reference nanoboxes. To exclude the influence of leached $\mathrm{Co}(\mathrm{CN})_{6}{ }^{3-}$ and $\mathrm{Fe}(\mathrm{CN})_{6}{ }^{3-}$, fresh samples of Co-Fe-S@PB NBs and Co-S@PB NBs were rinsed with $2 \mathrm{M} \mathrm{NaOH}$ (Figures S28 and S34). Both Co-S@PB NBs and Co-Fe-S@PB NBs showed negligible decline in their overpotential at $10 \mathrm{~mA} / \mathrm{cm}^{2}$ (Figures $5 \mathrm{~b}$-c). Importantly, without sulfidation the Co-PB and Co-Fe-PB NCs exhibited similar activity, which further suggested that the leached $(\mathrm{Co} / \mathrm{Fe})(\mathrm{CN})_{6}{ }^{3-}$ did not serve as OER active species, neither in Co-PB NCs and Co-S@PB NBs nor in Co-Fe-PB NCs and CoFe-S@PB NBs. Similar anionic leaching effects both in Co-PB and Co-Fe-PB NBs suggest that CoOOH was the real active species of Co-based PB under alkaline conditions.

The post-electrocatalytic Co K-edge in the XANES spectra of Co-Fe-S@PB NBs (Figure S35a) shows a positive edge shift, indicating an increase in the oxidation state of $\mathrm{Co}$. It should be pointed out that the line shape of the Co K-edge XANES spectra of the post-catalytic samples differs from the pristine, but is similar to the $\mathrm{Co}_{3} \mathrm{O}_{4}$ reference, suggesting that the electronic and local structures of cobalt ions of the post-catalytic material were changed and Co-based oxide species were formed in situ. The Fe K-edge XANES spectra provided analogous results to those obtained for Co (Figure S35b). The Fe K-edge XANES spectra of the post catalytic material showed a similar line shape to $\mathrm{Fe}_{2} \mathrm{O}_{3}$. These post catalytic results suggested that the catalytically active species of $\mathrm{Co}-\mathrm{Fe}-\mathrm{S} @ \mathrm{~PB} \mathrm{NBs}$ are in situ formed metal oxide species $\left.\left[(\mathrm{Co}, \mathrm{Fe}) \mathrm{O}_{\mathrm{x}},(\mathrm{Co}, \mathrm{Fe})(\mathrm{OH})_{\mathrm{y}}\right)\right]$, with higher OER activity compared to $\mathrm{CoO}_{\mathrm{x}}$ and $\mathrm{CoOOH} .{ }^{15-16}$ The latter is due to the fact that the active species of Co-PB NCs and Co-S@PB NBs, namely $\mathrm{CoO}_{\mathrm{x}}$ and $\mathrm{CoOOH}$, displayed a lower performance compared to the Co-Fe-S@PB NBs (Figure 5, Figures S27-29 and S31-34, and Supporting Information on CV tests). The in situ formation of metal oxide species was further confirmed by the post-catalytic XPS and TEM characterizations (Figures S36-39), which showed in their entirety that the boxlike nature of the catalysts was retained after electrochemical experiments and the leached $\mathrm{CoO}_{\mathrm{x}} / \mathrm{CoOOH}$ phases were the real active species of Co-S@PB NBs during OER. According to these leaching studies and post-catalytic studies (Figure 4, Figure S27-40, and Supplementary note on CV tests), the following conclusions were drawn (Figure S41): (1) Due to leaching processes of $(\mathrm{Co} / \mathrm{Fe})(\mathrm{CN})_{6}{ }^{3-}$ both in $\mathrm{Co}-\mathrm{PB}$ and $\mathrm{Co}-\mathrm{Fe}-$ PB NCs without the addition of sulfur, the actual active species were only related $\mathrm{Co}(\mathrm{OH})_{\mathrm{x}}$, so that similar activities toward OER were obtained in both sulfide-free Co-Fe-PB and Co-PB NCs. (2) During sulfidation, anion exchange processes occurred between $\mathrm{CN}^{-}$and $\mathrm{S}^{2-}$, leading to formation of the heterogeneous structures of Co-Fe-S@PB, and followed by further leaching of catalytically inactive $(\mathrm{Co} / \mathrm{Fe})(\mathrm{CN})_{6}^{3-}$ under electrochemical conditions. This partial formation of $(\mathrm{Co}, \mathrm{Fe}) \mathrm{S}_{\mathrm{x}}$ led to notably enhanced OER activity. (3) In contrast, $\mathrm{CoS}_{\mathrm{x}}$ prepared from CoPB nanocube precursors showed low activity compared to (Co, $\mathrm{Fe}) \mathrm{S}_{\mathrm{x}}$ due to a lack of synergistic effects between $\mathrm{Co}$ and $\mathrm{Fe} .{ }^{10-}$

${ }^{11}$ Previous DFT simulations for $\mathrm{Fe}$ in $\mathrm{Ni} / \mathrm{Co}$-based materials showed that $\mathrm{Fe}$ regulates the electronic structure and improves the redox activities of $\mathrm{Ni} / \mathrm{Co}$ catalysts. The activation energy of adsorption and desorption of intermediates is reduced, leading to the higher OER activity of Fe-containing catalysts..$^{16}$ (4) Postcatalytic characterizations including XANES, XPS and IIRSTEM (Figures S35-37) revealed the in situ formation of transition metal oxide species $\left[(\mathrm{Co}, \mathrm{Fe}) \mathrm{O}_{\mathrm{x}},(\mathrm{Co}, \mathrm{Fe})(\mathrm{OH})_{\mathrm{y}}\right]$ from the $\mathrm{Co}-\mathrm{Fe}-\mathrm{S} @ \mathrm{~PB} \mathrm{NBs}$, where these oxide species can serve as catalytically active sites for OER as reported in previous studies. ${ }^{58-62}$ Due to the unknown surface structure of the amorphous in situ formed products, we are currently working to address all the mechanistic steps of the above phenomena.

\section{Co-Fe-Mo-S double-layered nanoboxes as bifunctional electrocatalysts}

We furthermore applied our strategy for nanobox electrocatalyst preparation on a straightforward process to synthesize double-shelled NBs to construct a Co-Fe-Mo-S heterostructure as bifunctional electrocatalyst via nanocube assisted self-templating. Double-shelled Co-Fe-Mo-S NBs were obtained from Co-Fe-PB NC templates, after careful control of the experimental parameters upon introduction of $\left(\mathrm{NH}_{4}\right)_{2} \mathrm{MoS}_{4}$ in a second stage. PXRD patterns, EDX spectra and elemental analyses in Figure S42 and Table S5 confirmed that $\mathrm{Co}-\mathrm{Fe}-\mathrm{Mo}-\mathrm{S}$ containing NBs were successfully synthesized after treatment at $210^{\circ} \mathrm{C}$ for $24 \mathrm{~h}$. Figure 6 and Figure $\mathrm{S} 43$ show the well-defined hollow box morphology of the Co-Fe-Mo-S samples. STEM-EDX element mapping analysis revealed the uniform distribution of $\mathrm{S}$ inside and outside of the nanobox, while Mo was mainly distributed at the outer region. $\mathrm{Co}$ and $\mathrm{Fe}$ only accumulated within the nanobox, indicating the successful construction of $\mathrm{Co}-\mathrm{Fe}-\mathrm{Mo}-\mathrm{S}$ heterostructure nanoboxes.

The electrocatalytic OER and HER performance of the newly synthesized Co-Fe-Mo-S NBs was evaluated. Figure 7a shows the LSV curves of Co-Fe-Mo-S NBs, $\mathrm{MoS}_{2}$, and of a blank GC electrode in $1 \mathrm{M} \mathrm{PBS}$ and $1 \mathrm{M} \mathrm{KOH}$ towards OER. Obviously, $\mathrm{MoS}_{2}$ showed no OER activity regardless of the applied electrolyte, namely $1 \mathrm{M}$ PBS or $1 \mathrm{M} \mathrm{KOH}$. Enhanced OER performance was observed for the Co-Fe-Mo-S NBs, which verifies the advantages of their heterostructure features. A considerable OER performance with an overpotential of 303 $\mathrm{mV}$ at current densities of $10 \mathrm{~mA} / \mathrm{cm}^{2}$ and Tafel slope value of $42.06 \mathrm{mV} \mathrm{dec}^{-1}$ was achieved with $\mathrm{Co}-\mathrm{Fe}-\mathrm{Mo}-\mathrm{S}$ NBs as an anodic electrode under $1 \mathrm{M} \mathrm{KOH}$ conditions (Figure $7 \mathrm{a}$ and Figure S44). An overpotential of $516 \mathrm{mV}$ to acquire the current density at $10 \mathrm{~mA} / \mathrm{cm}^{2}$ was recorded in $1 \mathrm{M}$ PBS solution. The improved OER performance of Co-Fe-Mo-S NBs is comparable to many reported significant metal chalcogenide catalysts (Table S2). In addition, the electrochemical stability of Co-Fe-Mo-S NBs was tested in $1 \mathrm{M} \mathrm{KOH}$ solution and found to be excellent (Figures $7 \mathrm{~b}-\mathrm{c}$ ). Only a slight decline was observed in current density and LSV curves after $10000 \mathrm{~s}$, indicating the remarkable stability of Co-Fe-Mo-S NBs. The

Figure 6. FESEM and TEM images of as-prepared Co-Fe-PB NCs emerging from treatment with $\left(\mathrm{NH}_{3}\right)_{2} \mathrm{MoS}_{4}$ after different reaction times: (a, e) $2 \mathrm{~h},(\mathrm{~b}, \mathrm{f}) 12 \mathrm{~h},(\mathrm{c}, \mathrm{g}) 20 \mathrm{~h},(\mathrm{~d}, \mathrm{~h}) 24 \mathrm{~h}$, (i) HAADF-STEM and STEM-EDX elemental maps of as-prepared Co-Fe cubes after reaction for $20 \mathrm{~h}$ (Co: red, Fe: green, Mo: cyan, S: blue). 
Figure 7. (a) LSV curves with scan rate of $5 \mathrm{mV} / \mathrm{s}$ of Co-Fe-Mo-S NBs toward OER in $1 \mathrm{M}$ phosphate buffer solution PBS (pH 7.0) and 1 $\mathrm{M} \mathrm{KOH}$ (pH 13.6). (b) Time-dependent current density curves of Co-Fe-Mo-S in $1 \mathrm{M} \mathrm{KOH}$. (c) LSV curves of Co-Fe-Mo-S NBs before and

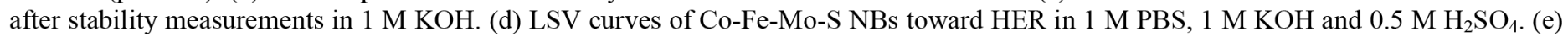
Time-dependent current density curves of Co-Fe-Mo-S NBs in $0.5 \mathrm{M} \mathrm{H}_{2} \mathrm{SO}_{4}$. (f) LSV curves of Co-Fe-Mo-S NBs before and after $12 \mathrm{~h}$ of stability measurements in $0.5 \mathrm{M} \mathrm{H}_{2} \mathrm{SO}_{4}$.

HER performance measurements in Figure $7 \mathrm{~d}$ and Figure S44b showed efficient HER activity of Co-Fe-Mo-S NBs throughout the $\mathrm{pH}$ scale. At the current density of $10 \mathrm{~mA} / \mathrm{cm}^{2}$, a small overpotential of $192 \mathrm{mV}$ with a Tafel slope value of $57.57 \mathrm{mV}$ $\mathrm{dec}^{-1}$ in $0.5 \mathrm{M} \mathrm{H}_{2} \mathrm{SO}_{4}, 300 \mathrm{mV}$ with $104.13 \mathrm{mV} \mathrm{dec}^{-1}$ in $1 \mathrm{M}$ $\mathrm{KOH}$, and $318 \mathrm{mV}$ with $130.77 \mathrm{mV} \mathrm{dec}^{-1}$ in $1 \mathrm{M}$ PBS were obtained which outperformed bare $\mathrm{MoS}_{2}$ and many of the previously reported materials such as $192 \mathrm{mV}$ for $\mathrm{CoS}_{2}, 210 \mathrm{mV}$ for $\mathrm{Co}_{3} \mathrm{~S}_{4} @ \mathrm{MoS}_{2}, 230 \mathrm{mV}$ for NiFe-N-CNT-rGO, $221 \mathrm{mV}$ for $\mathrm{CoP}, 240 \mathrm{mV}$ for $\mathrm{Co}_{9} \mathrm{~S}_{8} @ \mathrm{C}$ and other metal-sulfide materials (Table S6). Durability tests exhibited excellent stability of CoFe-Mo-S NBs in $0.5 \mathrm{M} \mathrm{H}_{2} \mathrm{SO}_{4}$ as shown in Figures 7e-f. Impressively, the as-prepared heterostructure was maintained at $20 \mathrm{~mA} / \mathrm{cm}^{2}$ for $12 \mathrm{~h}$ without any obvious performance degradation (Figure S45). At the end of these measurements, the post-catalytic LSV curve showed only a little negative shift compared to the initial one, corroborating further the good stability of the as-prepared Co-Fe-Mo-S NBs. From the results of STEM-EDX elemental mapping and XRD analyses in Figure 6 and Figure S42, we concluded that Co-Fe-Mo-S NBs exhibit a heterogeneous structure comprising of an outer layer of $\mathrm{MoS}_{2}$ and an inner layer of $\mathrm{Co}-\mathrm{Fe}-\mathrm{S}$. This unique structure offers a high surface area, leading to more exposed edge sites and shorter diffusion lengths of evolving gas molecules. Due to the synergistic mixture of three metal ions ( $\mathrm{Co}, \mathrm{Fe}$ and $\mathrm{Mo}$ ) we believe that the good catalytic activity and excellent properties of Co-Fe-Mo-S NBs arise from an improved electron transfer between adjoining metal-sulfide $(\mathrm{Co}, \mathrm{Fe})_{9} \mathrm{~S}_{8}$ phases and the $\mathrm{MoS}_{2}$ phase, which results in a high HER activity. The synergistic interactions with the in situ formed amorphous oxide layer on the surface of our catalyst can further enhance the OER performance, as reported in studies of Co-Fe-S $a$ PB. ${ }^{36-}$

37 Furthermore, the formation of vacancy sites on Mo atoms interacting with $\mathrm{Co}$ and/or $\mathrm{Fe}$ species result in charge imbalance, as previously reported for hollow $\mathrm{Co}_{9} \mathrm{~S}_{8}-\mathrm{MoS}_{2}$, $\mathrm{Co}_{3} \mathrm{~S}_{4}-\mathrm{MoS}_{2}$ and $\mathrm{CoS}_{2}-\mathrm{MoS}_{2}$ heterostructures. ${ }^{37-38,63}$ This could lead to strong electron donation from the Co-/Fe-species to Mo atoms through intermediate $\mathrm{S}$ atoms connected to the active CoFe and Mo centers. Similar phenomena have also been reported for sulfides containing $\mathrm{Fe}, \mathrm{Ni}$, and $\mathrm{Cu}$ species on $\mathrm{MoS}_{2}$ based catalysts. ${ }^{64-67}$ Moreover, the metal coordination and electronic structure in Co-Fe-Mo-S NBs increases the exchange current density of $\mathrm{MoS}_{2}$ and further boosts the synergetic effects between $\mathrm{Co}-\mathrm{Fe}$ and $\mathrm{Mo}$ at the interface region between $(\mathrm{Co}, \mathrm{Fe})_{9} \mathrm{~S}_{8}$ and $\mathrm{MoS}_{2}$ phases. The edge-terminated structure of $\mathrm{Co}-\mathrm{Fe}-\mathrm{Mo}-\mathrm{S}$ NBs could also render the edge sites more electroactive. ${ }^{34-35}$ We thus believe that the catalytic activity could be positively influenced by the increased contact surface area between $(\mathrm{Co}, \mathrm{Fe})_{9} \mathrm{~S}_{8}$ and $\mathrm{MoS}_{2}$ phases and the high surface area for electrochemical reactions of the Co-Fe-Mo-S NBs catalyst as shown in Figure S46. For definite conclusions on the underlying structure-activity relationships, follow-up in-situ characterizations and DFT-based computational studies will be essential. Further investigations into the electrochemical reaction mechanisms of the Co-Fe-Mo-S NBs are now under way to optimize their application potential.

\section{CONCLUSIONS}

Co-S@PB, Co-Fe-S@PB, and Ni-Fe-S@PB NB electrocatalysts were newly synthesized with a simple and robust self-templated strategy starting from their corresponding PB precursors. This facile approach can be tuned to convert the as-prepared precursors into two morphologies: Co-Fe-S@PB NBs and Co-Fe-S@PB particles. Co-Fe-S@PB NBs showed an excellent OER performance at a low overpotential of $286 \mathrm{mV}$ at the current density of $10 \mathrm{~mA} / \mathrm{cm}^{2}$ and outstanding electrochemical stability at a long operational time of $33 \mathrm{~h}$, thus outperforming the $\mathrm{RuO}_{2}$ standard.

The superior electrocatalytic performance of Co-Fe-S@PB is attributed to the following features: (1) The unique nanobox morphology enables full access to active sites, efficient electron transfer and robust chemical stability. (2) Synergistic effects through in situ formation of $\mathrm{Co} / \mathrm{Fe}$ oxide/hydroxides further enhance the electrocatalytic activity of Co-Fe-S@PB NBs. Investigation of the reaction pathways revealed the nature of leaching processes in Co-Fe-PB materials and showed that the main sulfidization route involves exchange between $\mathrm{S}^{2-}$ and $\mathrm{CN}^{-}$ from the released $\mathrm{Fe}(\mathrm{CN})_{6}{ }^{3-}$. As the surface structures of Co$\mathrm{S} @ \mathrm{~PB}, \mathrm{Co}-\mathrm{Fe}-\mathrm{S} @ \mathrm{~PB}, \mathrm{Ni}-\mathrm{Fe}-\mathrm{S} @ \mathrm{~PB}$ NBs, and their related in situ formed products consist of amorphous layers, more insight into the nature and catalytic changes of these amorphous surface structures must be extracted from in situ spectroscopic measurements. This will be essential for comprehensive DFTbased computational studies of the mechanistic processes in future works.

Importantly, the construction of Co-Fe-Mo-S heterostructure nanoboxes was achieved from further applications of our new self-templated approach. The as-prepared double-layered nanoboxes exhibited high activity in both OER and HER. Moreover, superior HER performance to pristine $\mathrm{MoS}_{2}$ was observed for a wide $\mathrm{pH}$ range between 0 and 14. This demonstrates the potential of our strategy to construct heterostructures of double-layered nanoboxes with excellent activity as bifunctional electrocatalysts.

Our technique is now ready to be applied on other key materials, such as selenides ${ }^{19-20}$ and phosphides, ${ }^{21-24}$ opening new pathways for other important electrochemical applications, such as $\mathrm{Na} / \mathrm{Li}$ ion storage $\mathrm{e}^{41-43}$ and $\mathrm{CO}_{2}$ reduction. ${ }^{68}$ This work paves the way to the targeted construction of hollow nanostructures with morphological benefits for energy conversion and storage devices.

Supporting Information. This material is available free of charge via the Internet at http://pubs.acs.org.

Details on the synthesis, analytical characterizations and electrocatalytic measurements can be found in the Supporting Information, together with additional XRD, SEM, EDX, HRSTEM, XPS and XAS data as well as the electrochemical properties of Co-S@PB, Co-Fe-S@PB, Ni-Fe-S@PB NBs, and post-catalytic Co-Fe-S@PB NBs, respectively. 


\section{AUTHOR INFORMATION}

\section{Corresponding Author}

* E-mail: greta.patzke@chem.uzh.ch.

\section{Author Contributions}

The manuscript was written through contributions of all authors. All authors have given approval to the final version of the manuscript.

\section{Notes}

The authors declare no competing financial interest.

\section{ACKNOWLEDGMENTS}

Y. G. Zhao, C. K. Mavrokefalos, J. G. Li, C. A. Triana and G. R. Patzke thank the University of Zurich and the UZH Research Priority Program "Solar Light to Chemical Energy Conversion" (URPP LightChEC) for financial support. G. R. Patzke thanks the Swiss National Science Foundation (Sinergia Grant No. CRSII2_160801) for financial support. R.E. acknowledges funding from the European Research Council (ERC) under EU's Horizon 2020 Research and Innovation Program (Grant agreement no. 681312). The authors acknowledge the assistance and support of the Center for Microscopy and Image Analysis (UZH) in performing scanning electron microscopy experiments. We thank ESRF Grenoble for the allocation of synchrotron radiation beamline and Dr. Hermann Emerich for providing assistance in using beamline BM-31.

\section{REFERENCES}

(1) Montoya, J. H.; Seitz, L. C.; Chakthranont, P.; Vojvodic, A.; Jaramillo, T. F.; Nørskov, J. K. Materials for Solar Fuels and Chemicals. Nat. Mater. 2017, 16, 70-81.

(2) Witman, M.; Ling, S.; Gladysiak, A.; Stylianou, K. C.; Smit, B.; Slater, B.; Haranczyk, M. Rational Design of a Low-Cost, HighPerformance Metal-Organic Framework for Hydrogen Storage and Carbon Capture. J. Phys. Chem. C 2017, 121, 1171-1181.

(3) Schäfer, H.; Kupper, K.; Schmidt, M.; Müller-Buschbaum, K.; Stangl, J.; Daum, D.; Steinhart, M.; Schulz-Kolbel, C.; Han, W.; Wollschlager, J.; Krupp, U.; Hou, P.; Liu, X. Steel-Based Electrocatalysts for Efficient and Durable Oxygen Evolution in Acidic Media. Catal. Sci. Technol. 2018, 8, 2104-2116.

(4) McCrory, C. C.; Jung, S., Peters, J. C.; Jaramillo, T. F. Benchmarking Heterogeneous Electrocatalysts for the Oxygen Evolution Reaction. J. Am. Chem. Soc. 2013, 135, 16977-16987.

(5) Duan, H.; Li, D.; Tang, Y.; He, Y.; Ji, S.; Wang, R.; Lv, H.; Lopes, P. P.; Paulikas, A. P.; Li, H.; Mao, S. X. High-Performance $\mathrm{Rh}_{2} \mathrm{P}$ Electrocatalyst for Efficient Water Splitting. J. Am. Chem. Soc. 2017, 139, 5494-5502.

(6) Burke, M. S.; Enman, L. J.; Batchellor, A. S.; Zou, S.; Boettcher, S. W. Oxygen Evolution Reaction Electro-catalysis on Transition Metal Oxides and (Oxy) Hydroxides: Activity Trends and Design Principles. Chem. Mater. 2015, 27, 7549-7558.

(7) Feng, Y.; Yu, X. Y.; Paik, U. Formation of $\mathrm{CO}_{3} \mathrm{O}_{4}$ Microframes from MOFs with Enhanced Electrochemical Performance for Lithium Storage and Water Oxidation. Chem. Commun. 2016, 52, 6269-6272.

(8) Wang, X.; Yu, L.; Guan, B. Y.; Song, S.; Lou, X. W. MetalOrganic Framework Hybrid-Assisted Formation of $\mathrm{Co}_{3} \mathrm{O}_{4} / \mathrm{Co}-\mathrm{Fe}$ Oxide Double-Shelled Nanoboxes for Enhanced Oxygen Evolution. Adv. Mater. 2018, 30, 1801211.

(9) Han, L.; Yu, X. Y.; Lou, X. W. Formation of Prussian-BlueAnalog Nanocages via a Direct Etching Method and their Conversion into Ni-Co-Mixed Oxide for Enhanced Oxygen Evolution. Adv. Mater. 2016, 28, 4601-4605.

(10) Zhuang, L.; Ge, L.; Yang, Y.; Li, M.; Jia, Y.; Yao, X.; Zhu, Z. Ultrathin Iron-Cobalt Oxide Nanosheets with Abundant Oxygen Vacancies for the Oxygen Evolution Reaction. Adv. Mater. 2017, 29, 1606793 .
(11) Ye, S. H.; Shi, Z. X.; Feng, J. X.; Tong, Y. X.; Li, G. R. Activating $\mathrm{CoOOH}$ Porous Nanosheet Arrays by Partial Iron Substitution for Efficient Oxygen Evolution Reaction. Angew. Chem. Int. Ed. 2018, 57, 2672-2676.

(12) Subbaraman, R.; Tripkovic, D.; Chang, K. C.; Strmenik, D.; Paulikas, A. P.; Hirunsit, P.; Chan, M.; Greeley, J.; Stamenkovic, V.; Markovic, N. M. Trends in Activity for the Water Electrolyser Reactions on $3 \mathrm{~d}$ M (Ni, Co, Fe, Mn) Hydr (Oxy) Oxide Catalysts. Nat. Mater. 2012, 11, 550-557.

(13) Huang, Z.-F.; Song, J.; Du, Y.; Xi, S.; Dou, S.; Nsanzimana, J. M. V.; Wang, C.; Xu, Z. J.; Wang, X. Chemical and Structural Origin of Lattice Oxygen Oxidation in Co-Zn Oxyhydroxide Oxygen Evolution Electrocatalysts. Nat. Energy 2019, 4, 329-338.

(14) Song, F.; Hu, X. Exfoliation of Layered Double Hydroxides for Enhanced Oxygen Evolution Catalysis. Nat. Commun. 2014, 5, 4477.

(15) Song, F.; Bai, L.; Moysiadou, A.; Lee, S.; Hu, C.; Liardet, L.; $\mathrm{Hu}, \mathrm{X}$. Transition Metal Oxides as Electrocatalysts for the Oxygen Evolution Reaction in Alkaline Solutions: An Application-Inspired Renaissance. J. Am. Chem. Soc. 2018, 140, 7748-7759.

(16) Burke, M. S.; Kast, M. G.; Trotochaud, L.; Smith, A. M.; Boettcher, S. W. Cobalt-Iron (Oxy) Hydroxide Oxygen Evolution Electrocatalysts: the Role of Structure and Composition on Activity, Stability, and Mechanism. J. Am. Chem. Soc. 2015, 137, 3638-3648.

(17) Dionigi, F.; Strasser, P. NiFe-Based (Oxy) Hydroxide Catalysts for Oxygen Evolution Reaction in Non-Acidic Electrolytes. Adv. Energy Mater. 2016, 6, 1600621.

(18) Yu, L.; Yang, J. F.; Guan, B. Y.; Lu, Y.; Lou, X. W. Hierarchical Hollow Nanoprisms Based on Ultrathin Ni-Fe Layered Double Hydroxide Nanosheets with Enhanced Electrocatalytic Activity towards Oxygen Evolution. Angew. Chem. Int. Ed. 2018, 57, 172-176.

(19) Xu, X.; Song, F.; Hu, X. A Nickel Iron Diselenide-Derived Efficient Oxygen-Evolution Catalyst. Nat. Commun. 2016, 7, 12324.

(20) Nai, J. W.; Lu, Y.; Yu, L.; Wang, X.; Lou, X. W. Formation of $\mathrm{Ni}-\mathrm{Fe}$ Mixed Diselenide Nanocages as a Superior Oxygen Evolution Electrocatalyst. Adv. Mater. 2017, 29, 1703870.

(21) Kim, J.; Jin, H.; Oh, A.; Baik, H.; Joo, S. H.; Lee, K. Synthesis of Compositionally Tunable, Hollow Mixed Metal Sulphide $\mathrm{Co}_{\mathrm{x}} \mathrm{Ni}_{\mathrm{y}} \mathrm{S}_{z}$ Octahedral Nanocages and Their Composition-Dependent Electrocatalytic Activities for Oxygen Evolution Reaction. Nanoscale 2017, 9, 15397-15406.

(22) Li, B. Q.; Zhang, S. Y.; Tang, C.; Cui, X.; Zhang, Q. Anionic Regulated NiFe (Oxy) Sulfide Electrocatalysts for Water Oxidation. Small 2017, 13, 1700610.

(23) Ahn, W.; Park, M. G.; Lee, D. U.; Seo, M. H.; Jiang, G.; Cano, Z. P.; Hassan, F. M.; Chen, Z. Hollow Multivoid Nanocuboids Derived from Ternary Ni-Co-Fe Prussian Blue Analog for DualElectrocatalysis of Oxygen and Hydrogen Evolution Reactions. $A d v$. Funct. Mater. 2018, 28, 1802129.

(24) Wang, C.; Wang, T.; Liu, J.; Zhou, Y.; Yu, D.; Han, F.; Li, Q.; Chen, J.; Huang, Y. Facile Synthesis of Silk-Cocoon S-Rich Cobalt Polysulfide as an Efficient Catalyst for the Hydrogen Evolution Reaction. Energy Environ. Sci. 2018, 11, 2467-2475.

(25) Xu, P.; Li, J.; Luo, J.; Wei, L.; Zhang, D.; Zhou, D.; Xu, W.; Yuan, D. $\left(\mathrm{Fe}_{0.2} \mathrm{Ni}_{0.8}\right)_{0.96} \mathrm{~S}$ Tubular Spheres Supported on Ni Foam as an Efficient Bifunctional Electrocatalyst for Overall Water Splitting. Sci. Rep. 2018, 8, 9425.

(26) Tang, C.; Gan, L.; Zhang, R.; Lu, W.; Jiang, X.; Asiri, A. M.; Sun, X.; Wang, J.; Chen, L. Ternary $\mathrm{Fe}_{\mathrm{x}} \mathrm{Co}_{1-\mathrm{x}} \mathrm{P}$ Nanowire Array as a Robust Hydrogen Evolution Reaction Electrocatalyst with Pt-like Activity: Experimental and Theoretical Insight. Nano Lett. 2016, 16, 6617-6621.

(27) Qiu, B.; Cai, L.; Wang, Y.; Lin, Z.; Zuo, Y.; Wang, M.; Chai, Y. Fabrication of Nickel-Cobalt Bimetal Phosphide Nanocages for Enhanced Oxygen Evolution Catalysis. Adv. Funct. Mater. 2018, 28, 1706008.

(28) Wu, R.; Xiao, B.; Gao, Q.; Zheng, Y. R.; Zheng, X. S.; Zhu, J. F.; Gao, M. R.; Yu, S. H. A Janus Nickel Cobalt Phosphide Catalyst for High-Efficiency Neutral-pH Water Splitting. Angew. Chem. Int. Ed. 2018, 57, 15671-15675. 
(29) Feng, Y.; Yu, X. Y.; Paik, U. Nickel Cobalt Phosphides QuasiHollow Nanocubes as an Efficient Electrocatalyst for Hydrogen Evolution in Alkaline Solution. Chem. Commun. 2016, 52, 1633-1636.

(30) Kampouri, S.; Nguyen, T. N.; Ireland, C. P.; Valizadeh, B.; Ebrahim, F. M.; Capano, G.; Ongari, D.; Mace, A.; Guijarro, N.; Sivula, K.; Sienkiewicz, A.; Forro, L.; Smit, B.; Stylianou, K. C. Photocatalytic Hydrogen Generation From a Visible-Light Responsive Metal-Organic Framework System: The Impact of Nickel Phosphide Nanoparticles. J. Mater. Chem. A 2018, 6, 2476-2481.

(31) Sheng, W.; Myint, M.; Chen, J. G.; Yan, Y. Correlating the Hydrogen Evolution Reaction Activity in Alkaline Electrolytes with the Hydrogen Binding Energy on Monometallic Surfaces. Energy Environ. Sci. 2013, 6, 1509-1512.

(32) Xie, J.; Zhang, J.; Li, S.; Grote, F.; Zhang, X.; Zhang, H.; Wang, R.; Lei, Y.; Pan, B.; Xie, Y. Controllable Disorder Engineering in Oxygen-Incorporated $\mathrm{MoS}_{2}$ Ultrathin Nanosheets for Efficient Hydrogen Evolution. J. Am. Chem. Soc. 2013, 135, 17881-17888.

(33) Huang, Z.-F.; Song, J.; Li, K.; Tahir, M.; Wang, Y.-T.; Pan, L.; Wang, L.; Zhang, X.; Zou, J.-J. Hollow Cobalt-Based Bimetallic Sulfide Polyhedra for Efficient All-pH Value Electrochemical and Photocatalytic Hydrogen Evolution. J. Am. Chem. Soc. 2016, 138, 1359-1365.

(34) Jaramillo, T. F.; Jørgensen, K. P.; Bonde, J.; Nielsen, J. H.; Horch, S.; Chorkendorff, I. Identification of Active Edge Sites for Electrochemical $\mathrm{H}_{2}$ Evolution from $\mathrm{MoS}_{2}$ Nanocatalysts. Science 2007, $317,100-102$.

(35) Lu, Z.; Zhu, W.; Yu, X.; Zhang, H.; Li, Y.; Sun, X.; Wang, X.; Wang, H.; Wang, J.; Luo, J.; Lei, X. Ultrahigh Hydrogen Evolution Performance of Under-Water "Superaerophobic" $\mathrm{MoS}_{2}$ Nanostructured Electrodes. Adv. Mater. 2014, 26, 2683-2687.

(36) Gao, M. R.; Liang, J. X.; Zheng, Y. R.; Xu, Y. F.; Jiang, J.; Gao, Q.; Li, J.; Yu, S. H. An Efficient Molybdenum Disulfide/Cobalt Diselenide Hybrid Catalyst for Electrochemical Hydrogen Generation. Nat. Commun. 2015, 6, 5982.

(37) Guo, Y.; Tang, J.; Qian, H.; Wang, Z.; Yamauchi, Y. One-Pot Synthesis of Zeolitic Imidazolate Framework 67-Derived Hollow $\mathrm{Co}_{3} \mathrm{~S}_{4} @ \mathrm{MoS}_{2}$ Heterostructures as Efficient Bifunctional Catalysts. Chem. Mater. 2017, 29, 5566-5573.

(38) Ramos, M.; Berhault, G.; Ferrer, D. A.; Torres, B.; Chianelli, R. R. HRTEM and Molecular Modeling of the $\mathrm{MoS}_{2}-\mathrm{Co}_{9} \mathrm{~S}_{8}$ Interface: Understanding the Promotion Effect in Bulk HDS Catalysts. Catal. Sci. Technol. 2012, 2, 164-178.

(39) Zhang, J.; Hu, Y.; Liu, D.; Yu, Y.; Zhang, B. Enhancing Oxygen Evolution Reaction at High Current Densities on AmorphousLike Ni-Fe-S Ultrathin Nanosheets via Oxygen Incorporation and Electrochemical Tuning. Adv. Sci. 2017, 4, 1600343.

(40) Zhuo, S.; Shi, Y.; Liu, L.; Li, R.; Shi, L.; Anjum, D. H.; Han, Y.; Wang, P. Dual-Template Engineering of Triple-Layered Nanoarray Electrode of Metal Chalcogenides Sandwiched with HydrogenSubstituted Graphdiyne. Nat. Commun. 2018, 9, 3132.

(41) Zhang, H.; Nai, J. W.; Yu, L.; Lou, X. W. D. Metal-OrganicFramework-Based Materials as Platforms for Renewable Energy and Environmental Applications. Joule 2017, 1, 77-107.

(42) Yu, L.; Yu, X. Y.; Lou, X. W. The Design and Synthesis of Hollow Micro-/Nanostructures: Present and Future Trends. Adv. Mater. 2018, 30, 1800939.

43) Nai, J. W.; Lou, X. W. Hollow Structures Based on Prussian Blue and Its Analogs for Electrochemical Energy Storage and Conversion. Adv. Mater. 2018, 1706825.

(44) Zhuo, S.; Xu, Y.; Zhao, W.; Zhang, J.; Zhang, B. Hierarchical Nanosheet-Based $\mathrm{MoS}_{2}$ Nanotubes Fabricated by an Anion-Exchange Reaction of $\mathrm{MoO}_{3}$-Amine Hybrid Nanowires. Angew. Chem. Int. Ed. 2013, 52, 8602-8606.

(45) Rieger, M.; Wittek, M.; Scherer, P.; Löbbecke, S.; MüllerBuschbaum, K. Preconcentration of Nitroalkanes with Archetype Metal-Organic Frameworks (MOFs) as Concept for a Sensitive Sensing of Explosives in the Gas Phase. Adv. Funct. Mater. 2018, 28, 1704250 .

(46) Chen, C.; Kang, Y.; Huo, Z.; Zhu, Z.; Huang, W.; Xin, H. L.; Snyder, J. D.; Li, D.; Herron, J. A.; Mavrikakis, M.; Chi, M. Highly
Crystalline Multimetallic Nanoframes with Three-Dimensional Electrocatalytic Surfaces. Science 2014, 343, 1339-1343.

(47) Pintado, S.; Goberna-Ferrón, S.; Escudero-Adán, E. C.; GalánMascarós, J. R. Fast and Persistent Electrocatalytic Water Oxidation by Co-Fe Prussian Blue Coordination Polymers. J. Am. Chem. Soc. 2013, 135, 13270-13273.

(48) Su, X.; Wang, Y.; Zhou, J.; Gu, S.; Li, J.; Zhang, S. Operando Spectroscopic Identification of Active Sites in NiFe Prussian Blue Analogues as Electrocatalysts: Activation of Oxygen Atoms for Oxygen Evolution Reaction. J. Am. Chem. Soc. 2018, 140, 1128611292 .

(49) Zhou, J.; Wang, Y.; Su, X.; Gu, S.; Liu, R.; Huang, Y.; Yan, S.; Li, J.; Zhang, S. Electrochemically Accessing Ultrathin Co (Oxy)Hydroxide Nanosheets and Operando Identifying Their Active Phase for the Oxygen Evolution Reaction. Energy Environ. Sci. 2019, 12, 739-746.

(50) Yu, Z. Y.; Duan, Y.; Liu, J. D.; Chen, Y.; Liu, X. K.; Liu, W.; Ma, T.; Li, Y.; Zheng, X. S.; Yao, T.; Gao, M. R. Unconventional CN Vacancies Suppress Iron-Leaching in Prussian Blue Analogue PreCatalyst for Boosted Oxygen Evolution Catalysis. Nat. Commun. 2019, 10, 2799.

(51) Cai, P.; Huang, J.; Chen, J.; Wen, Z. Oxygen-Containing Amorphous Cobalt Sulfide Porous Nanocubes as High-Activity Electrocatalysts for the Oxygen Evolution Reaction in an Alkaline/Neutral Medium. Angew. Chem. Int. Ed. 2017, 56, 48584861 .

(52) Guo, Y.; Tang, J.; Wang, Z.; Kang, Y. M.; Bando, Y.; Yamauchi, Y. Elaborately Assembled Core-Shell Structured Metal Sulfides as a Bifunctional Catalyst for Highly Efficient Electrochemical Overall Water Splitting. Nano Energy 2018, 47, 494502.

(53) Sato, O.; Iyoda, T.; Fujishima, A.; Hashimoto, K. Photoinduced Magnetization of a Cobalt-Iron Cyanide. Science 1996, 272, 704-705.

(54) Yokoyama, T.; Kiguchi, M.; Ohta, T.; Sato, O.; Einaga, Y.; Hashimoto, K. Local Structure of a Trapped Photoexcited State of a FeCo Cyanide Studied by X-Ray-Absorption Fine-Structure Spectroscopy. Phys. Rev. B 1999, 60, 9340-9446.

(55) Xiong, Q.; Wang, Y.; Liu, P. F.; Zheng, L. R.; Wang, G.; Yang, H. G.; Wong, P. K.; Zhang, H.; Zhao, H. Cobalt Covalent Doping in $\mathrm{MoS}_{2}$ to Induce Bifunctionality of Overall Water Splitting. Adv. Mater. 2018, 30, 1801450.

(56) Han, X.; Wu, X.; Deng, Y.; Liu, J.; Lu, J.; Zhong, C.; Hu, W. Ultrafine Pt Nanoparticle-Decorated Pyrite-Type $\mathrm{CoS}_{2}$ Nanosheet Arrays Coated on Carbon Cloth as a Bifunctional Electrode for Overall Water Splitting. Adv. Energy Mater. 2018, 8, 1800935.

(57) Suntivich, J.; May, K. J.; Gasteiger, H. A.; Goodenough, J. B.; Shao-Horn, Y. A Perovskite Oxide Optimized for Oxygen Evolution Catalysis from Molecular Orbital Principles. Science 2011, 334, 13831385.

(58) Fan, K.; Zou, H.; Lu, Y.; Chen, H.; Li, F.; Liu, J.; Sun, L.; Tong, L.; Toney, M. F.; Sui, M.; Yu, J. Direct Observation of Structural Evolution of Metal Chalcogenide in Electrocatalytic Water Oxidation. ACS Nano 2018, 12, 12369-12379.

(59) Seo, B.; Sa, Y. J.; Woo, J.; Kwon, K.; Park, J.; Shin, T. J.; Jeong, H. Y.; Joo, S. H. Size-Dependent Activity Trends Combined with in Situ X-Ray Absorption Spectroscopy Reveal Insights into Cobalt Oxide/Carbon Nanotube-Catalyzed Bifunctional Oxygen Electrocatalysis. ACS Catal, 2016, 6, 4347-4355.

(60) Liu, Y.; Li, Q.; Si, R.; Li, G. D.; Li, W.; Liu, D. P.; Wang, D.; Sun, L.; Zhang, Y.; Zou, X. Coupling Sub-Nanometric Copper Clusters with Quasi-Amorphous Cobalt Sulfide Yields Efficient and Robust Electrocatalysts for Water Splitting Reaction. Adv. Mater. 2017, 29, 1606200

(61) Hu, C.; Zhang, L.; Zhao, Z. J.; Li, A.; Chang, X.; Gong, J. Synergism of Geometric Construction and Electronic Regulation: 3D Se- $(\mathrm{NiCo}) \mathrm{S}_{\mathrm{x}} /(\mathrm{OH})_{\mathrm{x}}$ Nanosheets for Highly Efficient Overall Water Splitting. Adv. Mater. 2018, 30, 1705538.

(62) Fang, L.; Li, W.; Guan, Y.; Feng, Y.; Zhang, H.; Wang, S.; Wang, Y. Tuning Unique Peapod-Like $\mathrm{Co}\left(\mathrm{S}_{\mathrm{x}} \mathrm{Se}_{1-\mathrm{x}}\right)_{2}$ Nanoparticles for Efficient Overall Water Splitting. Adv. Funct. Mater. 2017, 27, 1701008 . 
(63) Zhang, H.; Li, Y.; Xu, T.; Wang, J.; Huo, Z.; Wan, P.; Sun, X. Amorphous Co-doped $\mathrm{MoS}_{2}$ nanosheet coated metallic $\mathrm{CoS}_{2}$ nanocubes as an excellent electrocatalyst for hydrogen evolution. $J$. Mater. Chem. A. 2015, 3, 15020-15023.

(64) Yu, X. Y.; Feng, Y.; Jeon, Y.; Guan, B.; Lou, X. W.; Paik, U. Formation of Ni-Co-MoS 2 nanoboxes with enhanced electrocatalytic activity for hydrogen evolution. Adv. Mater. 2016, 28, 9006-9011.

(65) Wang, H.; Tsai, G.; Kong, D.; Chan, K.; Abild-Pederson, F.; Norskov, J. K.; Gui Y. Transition-metal doped edge sites in vertically aligned $\mathrm{MoS}_{2}$ catalysts for enhanced hydrogen evolution. Nano Res. $\mathbf{2 0 1 5}, 8$ (2), 566-575.
(66) Wang, D.; Zhang, X.; Shen, Y.; Wu, Z. Ni-doped $\mathrm{MoS}_{2}$ nanoparticles as highly active hydrogen evolution electrocatalysts. $R S C A d v$. 2016, 6, 16656-16661.

(67) Cui, W.; Ge, C.; Xing, Z.; Asiri, A. M.; Sun, X. Ni $\mathrm{S}_{\mathrm{y}}-\mathrm{MoS}_{2}$ hybrid microspheres: one-pot hydrothermal synthesis and their application as a novel hydrogen evolution reaction electrocatalyst with enhanced activity. Electrochim. Acta. 2014, 137, 504-510.

(68) Wang, S.; Guan, B. Y.; Lou, X. W. D. Construction of $\mathrm{ZnIn}_{2} \mathrm{~S}_{4}$ $\mathrm{In}_{2} \mathrm{O}_{3}$ Hierarchical Tubular Heterostructures for Efficient $\mathrm{CO}_{2}$ Photoreduction. J. Am. Chem. Soc. 2018, 140, 5037-5040. 


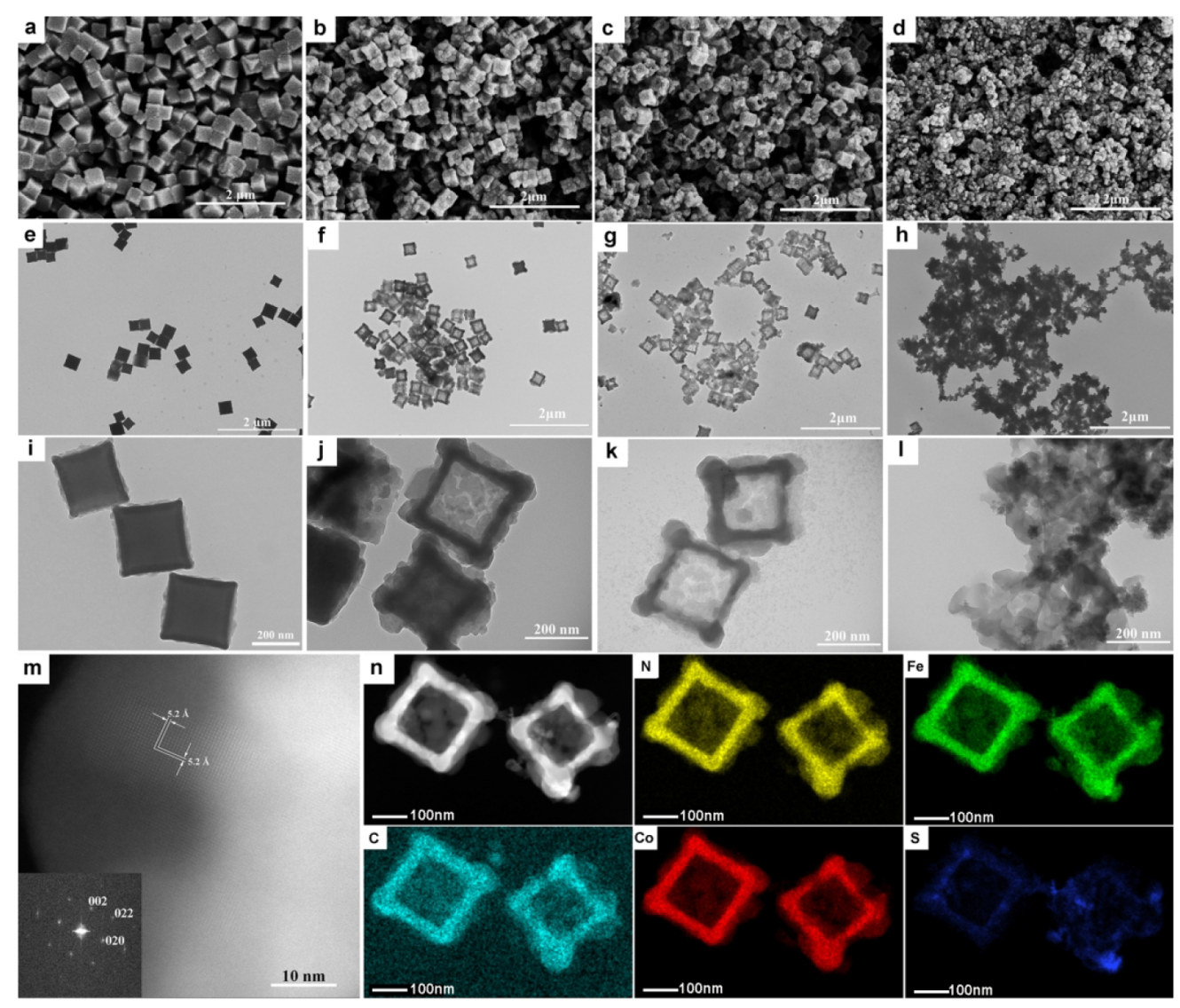

Figure 1 

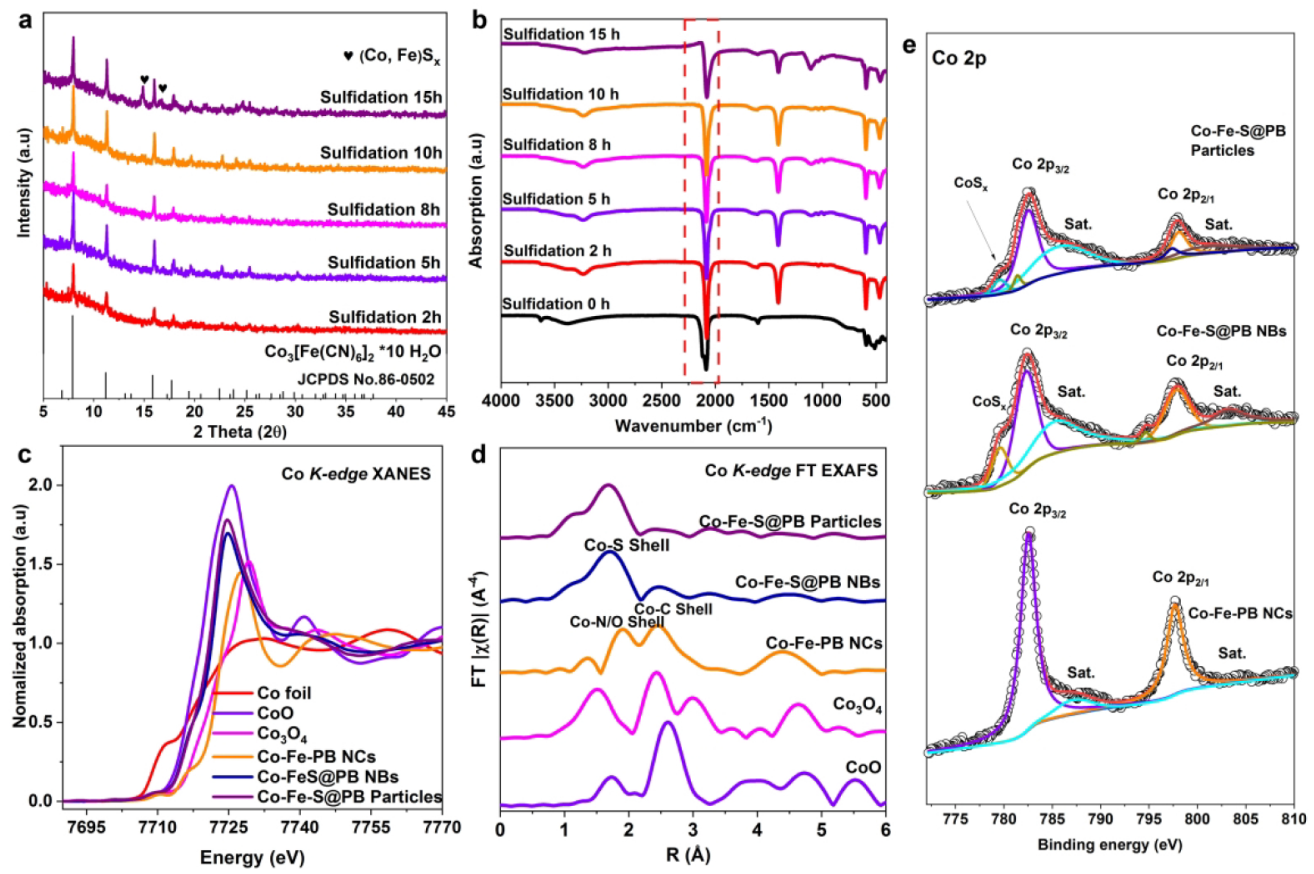

Figure 2 

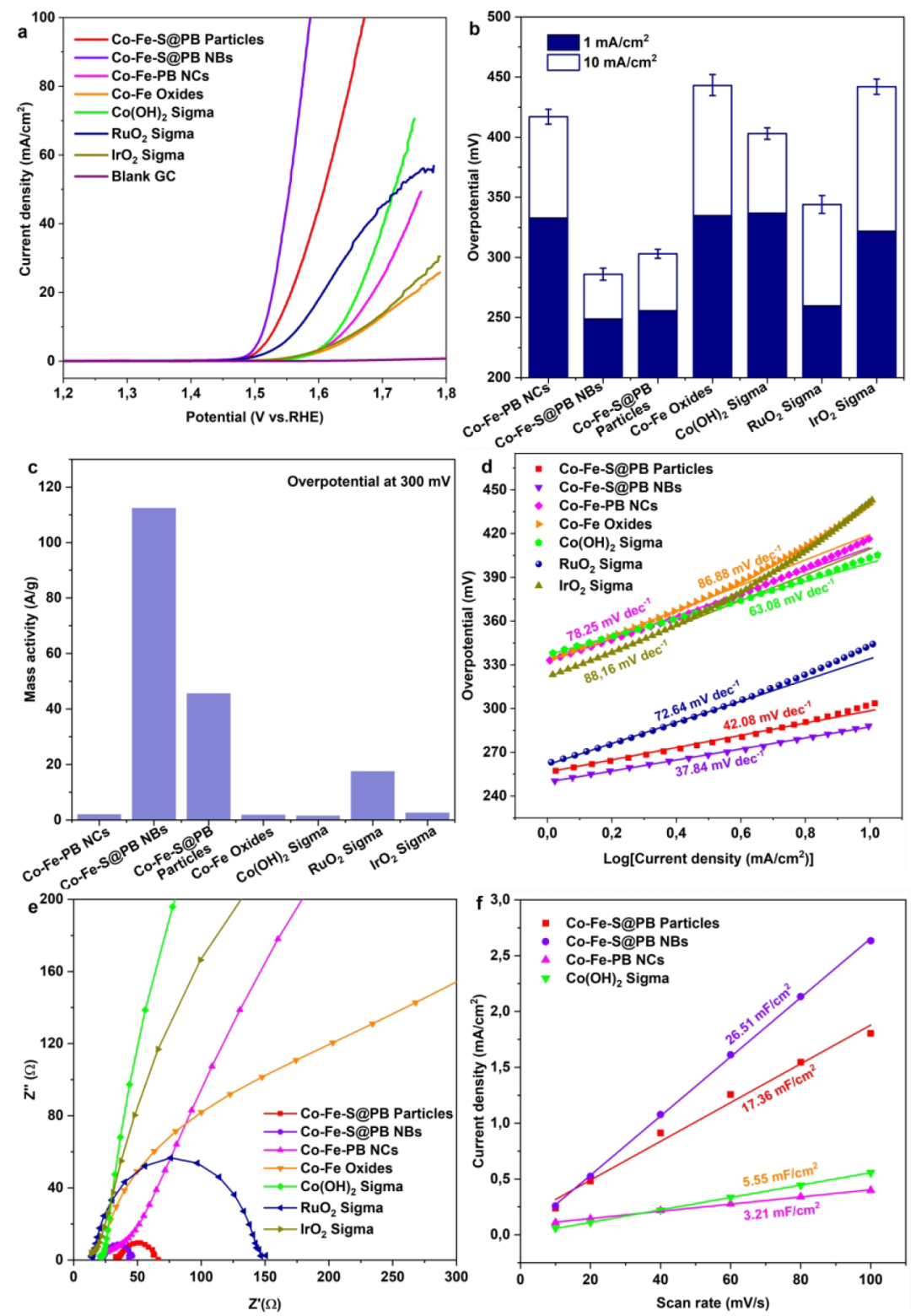

Figure 3 

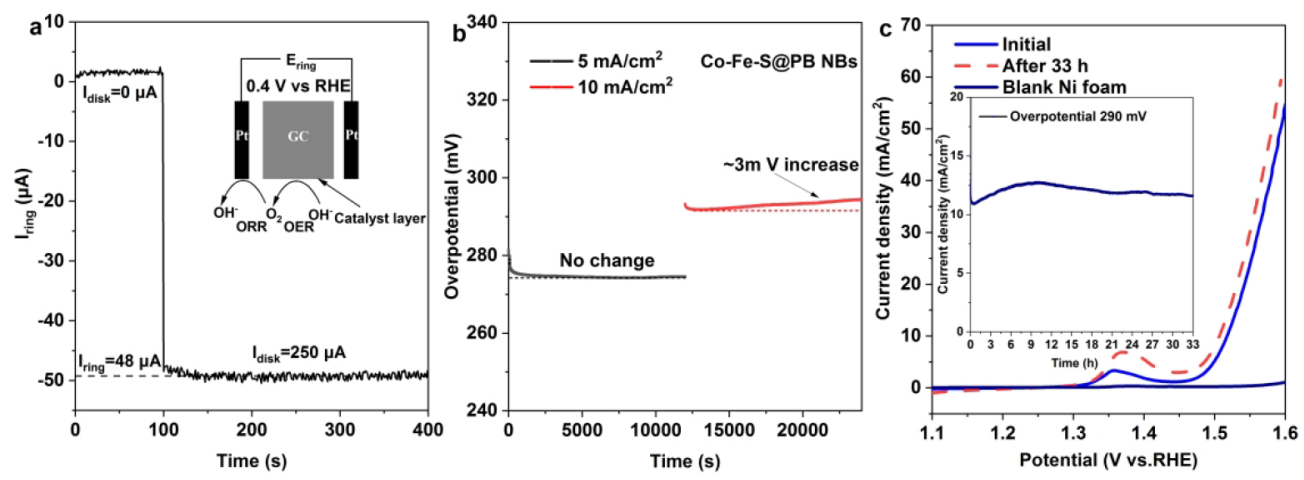

Figure 4 

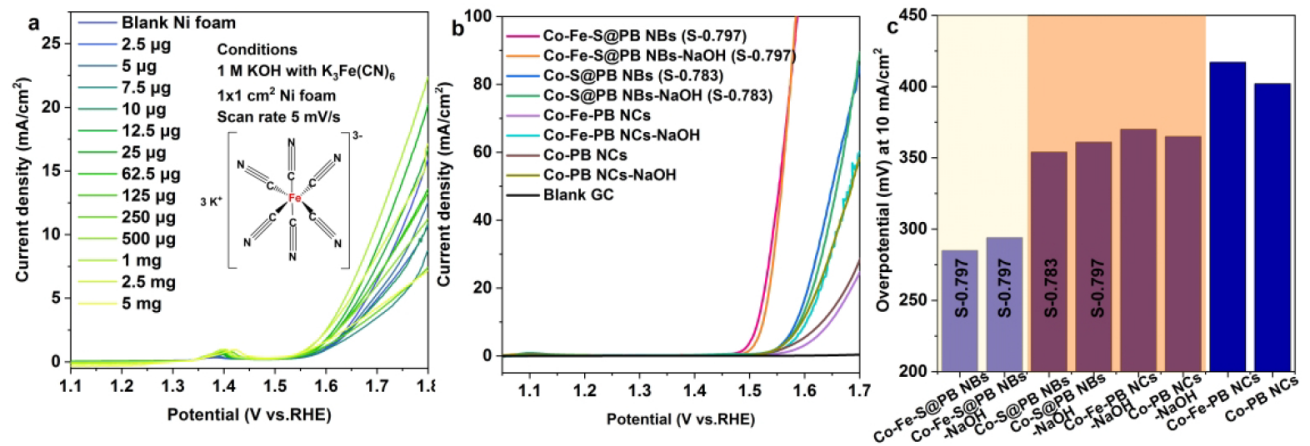

Figure 5 


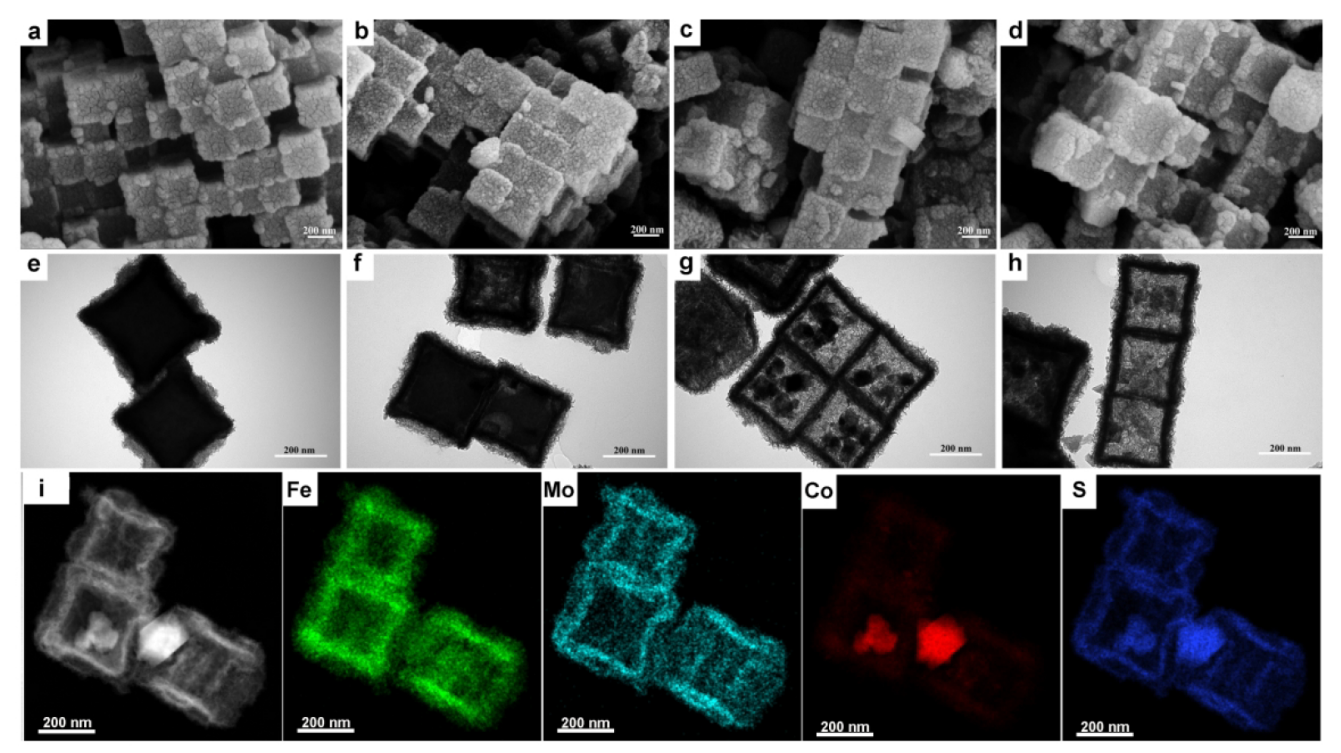

Figure 6 

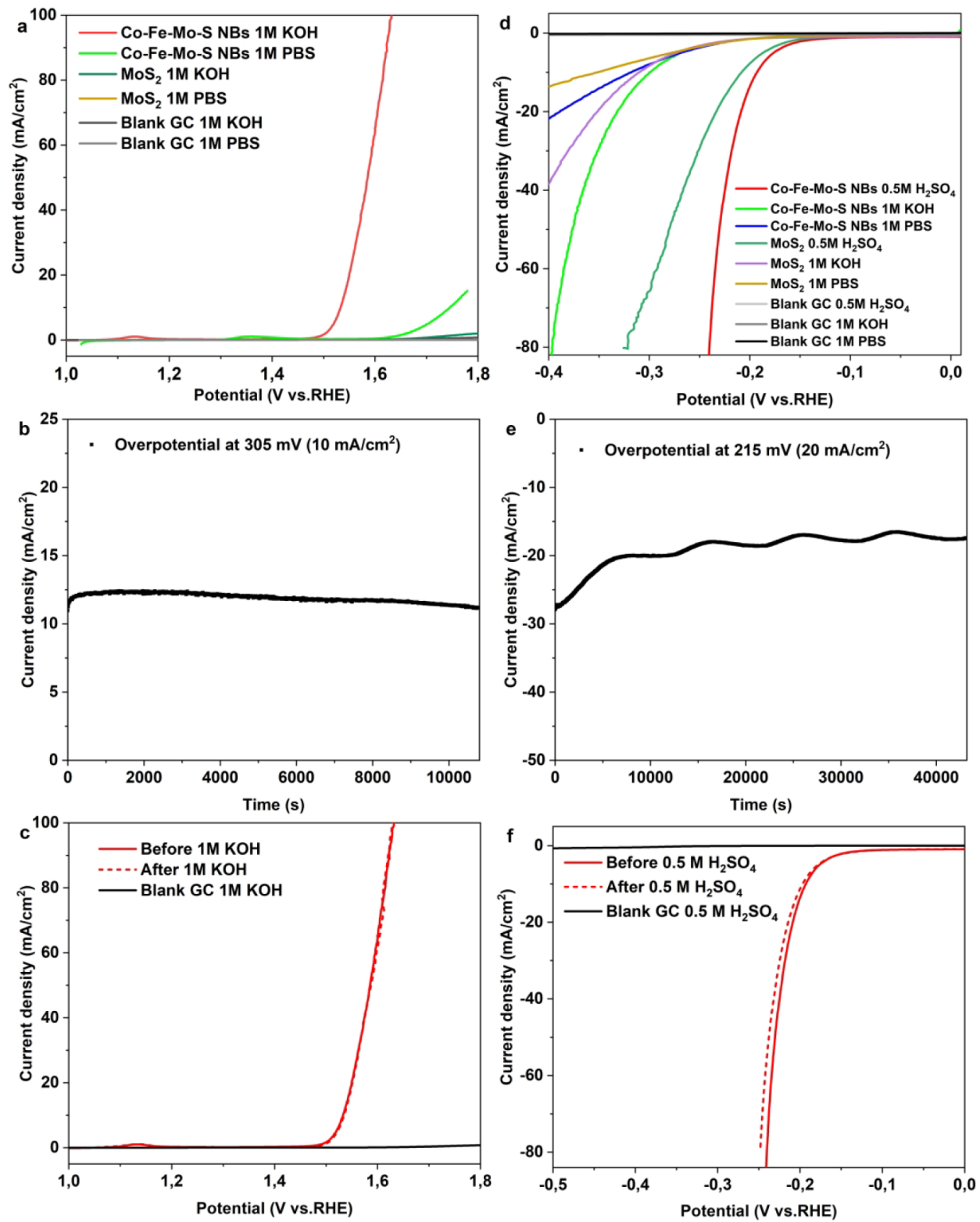

Figure 7 